\title{
HYPERASYMPTOTIC SOLUTIONS OF SECOND-ORDER LINEAR DIFFERENTIAL EQUATIONS I
}

\author{
A. B. Olde Daalhuis and F. W. J. Olver
}

\begin{abstract}
A sequence of re-expansions is developed for the remainder terms in the well-known Poincaré series expansions of the solutions of homogeneous linear differential equations of the second order in the neighborhood of an irregular singularity of rank one. These re-expansions are in series of repeated integrals of the generalized exponential integral, and the coefficients in these series are the same as those of the original Poincaré expansions. Each step of the process reduces the estimate of the error term by the same exponentially-small factor, while increasing the region of validity.

It also is shown how to ensure that the process is numerically stable. A numerical example is included.
\end{abstract}

\section{Introduction and summary}

The general homogeneous linear differential equation of the second order is given by

$$
\frac{d^{2} w}{d z^{2}}+f(z) \frac{d w}{d z}+g(z) w=0 .
$$

If, as we shall suppose, the point at infinity is an irregular singularity of rank one, then the functions $f(z)$ and $g(z)$ can be expanded in power series

$$
f(z)=\sum_{s=0}^{\infty} \frac{f_{s}}{z^{s}}, \quad g(z)=\sum_{s=0}^{\infty} \frac{g_{s}}{z^{s}},
$$

that converge on an open annulus $|z|>a$. Not all of the coefficients $f_{0}, g_{0}$, and $g_{1}$ vanish, otherwise infinity would be a regular singularity.

In a recent paper [6], we showed how to improve the accuracy and extend the region of applicability of the well-known Poincaré asymptotic expansions of the solutions of (1.1) for large $|z|$. This was achieved by truncating the expansions at or near their optimal stage (that is, as a rule, at or near their smallest term) and re-expanding the remainder term in a series of generalized exponential integrals. In the present paper, we show how to achieve additional improvement, both in accuracy and in the region of validity, by further re-expansions of remainder terms.

We follow existing terminology $[1,2,5,9]$ for these new types of asymptotic expansions. Thus re-expansions in terms of generalized exponential integrals are called

Received June 2, 1994, revised November 3, 1994

1991 Mathematics Subject Classification. Primary 34E05; Secondary 33B20, 33C10.

Key words and phrases: asymptotic expansions, Bessel functions, connection formulas, differential equations, exponential improvement, exponential integral, hyperasymptotics, hyperterminant, incomplete Gamma function, irregular singularity, remainder term, superasymptotics.

The research of the second author was supported by the National Science Foundation under Grant DMS 92-08690. 
exponentially-improved or superasymptotic expansions. Further re-expansions are called hyperasymptotic expansions. The levels of improvement are enumerated in the following way. The original Poincaré expansions are regarded as being at level zero, the exponentially-improved expansions are at level one, and the hyperasymptotic expansions are at levels two and above.

The paper is arranged as follows. In $\S 2$, we define and describe properties of certain multiple integrals, the so-called "hyperterminants", that are needed in subsequent sections. In $\S \S 3,4$, we show how to optimize the remainder terms in the well-known Poincaré or "zeroth-level" asymptotic expansions for the solutions of the second-order linear differential equation in the neighborhood of an irregular singularity of rank one. This problem is well understood in the literature, but we introduce a somewhat different form of proof that is to be used in later sections.

In $\S 5$, we re-expand the remainder terms that appear in $\S 4$, to obtain "first-level" expansions. These expansions are in series of generalized exponential integrals, or firstlevel hyperterminants, and are the same as those found in [6]. However, we now are able to optimize this re-expansion and the outcome is perhaps somewhat surprising. By taking approximately $2|z|$ terms in the zeroth-level expansion - that is, twice the number used when we optimize at that level - and $|z|$ terms in the first-level expansion, the error estimate is improved from $e^{-|z|}$ times an arbitrary power of $z^{-1}$ to $e^{-2|z|}|z|^{-\frac{1}{2}}$.

In $\S \S 6,7$, the process of re-expansion is continued to the second level and then generalized. At the $l$ th level, $l$ being arbitrary, the expansions of the solutions are in terms of hyperterminants at that level and all preceding levels, and the coefficients at each level alternate between the sets of coefficients for the original pair of Poincaré expansions. On optimizing, we find that the pattern described in the preceding paragraph continues. Thus we require approximately $(l+1)|z|$ terms at level zero, $l|z|$ terms at level one, and so on, finishing with $|z|$ terms at level $l$. The total number of terms is approximately $\frac{1}{2}(l+1)(l+2)|z|$, and the final error estimate is $e^{-(l+1)|z|}$ times a power of $z$. Since $l$ is arbitrary, this means that, in theory, there is no limit on the attainable exponential improvement.

In $\S 8$, the numerical stability of the generalized asymptotic expansion is examined, and it is demonstrated that instability, in the form of cancellation, begins when level 2 is included, and becomes severe at higher levels. It also is shown how to overcome this difficulty by reducing the number of terms called for in the optimization process that is used in $\S 7$. With the numerically stable form of expansion, the optimal error estimate on stopping at the $l$ th level $(l \geqslant 2)$ is $(l+1)^{-e|z|}$ times a power of $z$. The number of terms required also is less.

In $\S 9$, a numerical example is provided. In $\S 10$, it is shown how to extend the sector of validity beyond $|\mathrm{ph} z| \leqslant \pi$ for the hyperasymptotic expansions of $\S 7$. This is achieved via continuation formulas for the remainder terms. The sector of validity can extend as far as $|\operatorname{ph} z| \leqslant\left(l+\frac{3}{2}\right) \pi-\delta$, but as the sector widens the exponential improvement diminishes. A similar, but less extensive, continuation can also be made for the numerically stable hyperasymptotic expansions of $\S 8$.

In the concluding section, $\S 11$, comparisons of our new results are made with earlier results obtained in an important paper by Berry and Howls [1]. Comments also are made on the Stokes phenomenon and the numerical evaluation of the hyperterminants. 


\section{Auxiliary functions}

In [6] our re-expansions for the solutions of (1.1) were expressed in terms of the function $F_{p}(z)$ defined by

$$
F_{p}(z)=\frac{e^{-z}}{2 \pi} \int_{0}^{\infty} \frac{e^{-z t} t^{p-1}}{1+t} d t
$$

when $\Re p>0$ and $|\mathrm{ph} z|<\frac{1}{2} \pi$, and by analytic continuation elsewhere. This function is directly related to the generalized exponential integral and the incomplete Gamma function, and its relevant properties are summarized in $\S 2$ of [9].

In the present paper, we shall need the following generalizations of $F_{p}(z)$ :

$$
\begin{gathered}
G_{\omega}^{(0)}(z)=\frac{1}{z} \\
G_{\omega}^{(1)}\left(z ; M_{0}\right)=\int_{0}^{\infty} \frac{e^{-t} t^{M_{0}-1+\omega}}{t+z} d t
\end{gathered}
$$

and, in general,

$$
\begin{aligned}
& G_{\omega}^{(l)}\left(z ; M_{0}, M_{1}, \ldots, M_{l-1}\right)= \\
& \quad \int_{0}^{\infty} \cdots \int_{0}^{\infty} \frac{e^{-t-t_{1}-\cdots-t_{l-1}} t^{M_{0}+\omega} t_{1}^{M_{1}-\omega} \cdots t_{l-2}^{M_{l-2}+(-)^{l-2} \omega} t_{l-1}^{M_{l-1}-1+(-)^{l-1} \omega}}{(t+z)\left(t_{1}+t\right)\left(t_{2}+t_{1}\right) \cdots\left(t_{l-1}+t_{l-2}\right)} d t_{l-1} \cdots d t_{1} d t .
\end{aligned}
$$

Here, $z$ and $\omega$ are real or complex parameters and $l, M_{0}, M_{1}, \ldots$ are nonnegative integers. By observing that

$$
t_{1}+t \geqslant t, \quad t_{j}+t_{j-1} \geqslant t_{j-1}, \quad 2 \leq j<l-1,
$$

we see that these multiple integrals converge when $|\mathrm{ph} z|<\pi$ and

$$
M_{j}+(-)^{j} \Re \omega>0, \quad 0 \leq j<l-1 \text {. }
$$

Elsewhere, the $G_{\omega}^{(l)}$ are defined by analytic continuation with respect to $z$ and $\omega$. Clearly, when (2.4) applies the only singularities of $G_{\omega}^{(l)}\left(z ; M_{0}, \ldots, M_{l-1}\right)$ are branchpoints or poles at $z=0$ and $\infty$.

It will be observed that there is some degree of arbitrariness in the choice of the parameter $\omega$ and the integers $M_{0}, M_{1}, \ldots, M_{l-1}$. For example, from (2.1) we see that

$$
F_{M_{0}+\omega}(z)=(2 \pi)^{-1} e^{-z} z^{1-M_{0}-\omega} G_{\omega}^{(1)}\left(z ; M_{0}\right),
$$

but we equally well could write

$$
F_{M_{0}+\omega}(z)=(2 \pi)^{-1} e^{-z} z^{1-M_{0}-\omega} G_{\omega-1}^{(1)}\left(z ; M_{0}+1\right) .
$$

In the present paper, this ambiguity is resolved by requiring that $\omega$ is always determined by

$$
\omega=\mu_{2}-\mu_{1}
$$


where $\mu_{1}$ and $\mu_{2}$ are defined in the next section. The functions $G_{\omega}^{(l)}$ are closely related to the "generalized terminants" or "hyperterminants" introduced in $[1,2]$, and some properties of the $G_{\omega}^{(l)}$ follow directly from those of the $K_{r n}$ given in [1]. Other properties are as follows.

From (2.2) we have immediately

$$
G_{\omega}^{(l)}\left(z ; M_{0}, \ldots, M_{l-1}\right)=\int_{0}^{\infty} \frac{e^{-t} t^{M_{0}+\omega}}{t+z} G_{-\omega}^{(l-1)}\left(t ; M_{1}, \ldots, M_{l-1}\right) d t,
$$

when $l \geqslant 1$, and hence

$$
G_{\omega}^{(l)}\left(z ; M_{0}, \ldots, M_{l-1}\right)=z^{M_{l,}+\omega} \int_{0}^{\infty} \frac{e^{-z t} t^{M_{0}+\omega}}{t+1} G_{-\omega}^{(l-1)}\left(z t ; M_{1}, \ldots, M_{l-1}\right) d t,
$$

provided that $|\mathrm{ph} z|<\frac{1}{2} \pi$. If we rotate the path in this integral through an angle $2 \pi$ and subsequently replace $z$ by $z e^{-2 \pi i}$, then we obtain the continuation formula

$$
\begin{aligned}
G_{\omega}^{(l)}\left(z e^{-2 \pi i}\right. & \left.; M_{0}, \ldots, M_{l-1}\right)-G_{\omega}^{(l)}\left(z ; M_{0}, \ldots, M_{l-1}\right) \\
& =2 \pi i e^{-\left(M_{0}+\omega\right) \pi i} e^{z} z^{M_{0}+\omega} G_{-\omega}^{(l-1)}\left(z e^{-\pi i} ; M_{1}, \ldots, M_{l-1}\right),
\end{aligned}
$$

again valid when $l \geqslant 1$.

An upper bound for $G_{\omega}^{(l)}$ can be found from $(2.2 c)$ by use of (2.3) and the inequality

$$
|t+z| \geqslant|z| \mathcal{S}(z)
$$

where $\mathcal{S}(z)=1$ if $|\operatorname{ph} z| \leqslant \frac{1}{2} \pi$, and $\mathcal{S}(z)=|\sin (\operatorname{ph} z)|$ if $\frac{1}{2} \pi \leqslant|\operatorname{ph} z|<\pi$. Again, the integrals uncouple and we find that

$$
\left|G_{\omega}^{(l)}\left(z ; M_{0}, \ldots, M_{l-1}\right)\right| \leqslant \frac{\Gamma\left(M_{0}+\Re \omega\right) \Gamma\left(M_{1}-\Re \omega\right) \cdots \Gamma\left(M_{l-1}+(-)^{l-1} \Re \omega\right)}{|z| \mathcal{S}(z)}
$$

valid when $|\mathrm{ph} z|<\pi$ and (2.4) applies.

For the closed sector $|\mathrm{ph} z| \leqslant \pi$ we give the following estimate.

Lemma 2.1. If $M_{0}=|z|+\mathcal{O}(1)$, then as $z \rightarrow \infty$ in $|\mathrm{ph} z| \leqslant \pi$

$$
\begin{aligned}
& G_{\omega}^{(l)}\left(z ; M_{0}, \ldots, M_{l-1}\right) \\
& \quad=\Gamma\left(M_{0}+\Re \omega\right) \Gamma\left(M_{1}-\Re \omega\right) \cdots \Gamma\left(M_{l-1}+(-)^{l-1} \Re \omega\right) \mathcal{O}\left(z^{-\frac{1}{2}}\right),
\end{aligned}
$$

uniformly for all positive integers $l$.

Proof. Let $\delta$ be a constant such that $0<\delta<\pi$. If $|\operatorname{ph} z| \leqslant \pi-\delta$, then $\mathcal{S}(z) \geqslant \sin \delta$, and (2.12) is simply a weaker form of (2.11).

Next, in consequence of $(2.5 \mathrm{a})$ and $[9,(2.9)]$, we know that

$$
G_{\omega}^{(1)}\left(z ; M_{0}\right)=\mathcal{O}\left(e^{-|z|} z^{M_{0}-1+\Re \omega}\right)=\Gamma\left(M_{0}+\Re \omega\right) \mathcal{O}\left(z^{-\frac{1}{2}}\right),
$$

which proves (2.12) for $l=1$.

Now suppose that $l \geqslant 2$ and $0<\operatorname{ph} z<\pi$. On substituting into $(2.2 \mathrm{c})$ by means of the identity

$$
\frac{1}{(t+z)\left(t_{1}+t\right)}=\frac{1}{(t+z)\left(t_{1}+e^{-\pi i} z\right)}-\frac{1}{\left(t_{1}+e^{-\pi i} z\right)\left(t_{1}+t\right)}
$$


and using $(2.2 b)$, we obtain

$$
\begin{aligned}
& G_{\omega}^{(l)}\left(z ; M_{0}, \ldots, M_{l-1}\right)=G_{\omega}^{(1)}\left(z ; M_{0}+1\right) G_{-\omega}^{(l-1)}\left(e^{-\pi i} z ; M_{1}, \ldots, M_{l-1}\right) \\
& \quad-\int_{0}^{\infty} \cdots \int_{0}^{\infty} \frac{e^{-t-t_{1}-\cdots-t_{l-1}} t^{M_{0}+\omega} t_{1}^{M_{1}-\omega} \cdots t_{l-1}^{M_{l-1}-1+(-)^{l-1} \omega}}{\left(t_{1}+e^{-\pi i} z\right)\left(t_{1}+t\right)\left(t_{2}+t_{1}\right) \cdots\left(t_{l-1}+t_{l-2}\right)} d t_{l-1} \cdots d t_{1} d t .
\end{aligned}
$$

Analytic continuation extends the validity of this result to the sector $0<\operatorname{ph} z<2 \pi$. If we restrict $\delta \leqslant \mathrm{ph} z \leqslant \pi$, then from (2.11) and (2.13) we obtain

$$
\begin{aligned}
G_{\omega}^{(1)} & \left(z ; M_{0}+1\right) G_{-\omega}^{(l-1)}\left(e^{-\pi i} z ; M_{1}, \ldots, M_{l-1}\right) \\
& =\frac{\Gamma\left(M_{0}+1+\Re \omega\right) \Gamma\left(M_{1}-\Re \omega\right) \cdots \Gamma\left(M_{l-1}+(-)^{l-1} \Re \omega\right)}{z} \mathcal{O}\left(z^{-\frac{1}{2}}\right) \\
& =\Gamma\left(M_{0}+\Re \omega\right) \Gamma\left(M_{1}-\Re \omega\right) \cdots \Gamma\left(M_{l-1}+(-)^{l-1} \Re \omega\right) \mathcal{O}\left(z^{-\frac{1}{2}}\right) .
\end{aligned}
$$

The multiple integral in (2.15) may be estimated by analysis similar to that used to establish (2.11). We find that

$$
\begin{aligned}
\int_{0}^{\infty} \cdots \int_{0}^{\infty} \frac{e^{-t-t_{1}-\cdots-t_{l-1}} t^{M_{0}+\omega} t_{1}^{M_{1}-\omega} \cdots t_{l-1}^{M_{l-1}-1+(-)^{l-1} \omega}}{\left(t_{1}+e^{-\pi i} z\right)\left(t_{1}+t\right)\left(t_{2}+t_{1}\right) \cdots\left(t_{l-1}+t_{l-2}\right)} d t_{l-1} \cdots d t_{1} d t \\
\quad=\Gamma\left(M_{0}+\Re \omega\right) \Gamma\left(M_{1}-\Re \omega\right) \cdots \Gamma\left(M_{l-1}+(-)^{l-1} \Re \omega\right) \mathcal{O}\left(z^{-1}\right)
\end{aligned}
$$

as $z \rightarrow \infty$ in $\delta \leqslant \operatorname{ph} z \leqslant 2 \pi-\delta$.

On substituting into (2.15) by means of the last two equations, we obtain (2.12) for $\delta \leqslant \operatorname{ph} z \leqslant \pi$. The proof of (2.12) for the sector $-\pi \leqslant \operatorname{ph} z \leqslant-\delta$ is similar.

\section{Integral representations for the original remainder terms}

As in [6], we may assume, without loss of generality, that the roots $\lambda_{1}, \lambda_{2}$ of the characteristic equation

$$
\lambda^{2}+f_{0} \lambda+g_{0}=0
$$

satisfy

$$
\lambda_{2}-\lambda_{1}=1
$$

Then equation (1.1) has unique solutions $w_{1}(z)$ and $w_{2}(z)$ such that as $|z| \rightarrow \infty$

$$
\begin{gathered}
w_{1}(z) \sim e^{\lambda_{1} z} z^{\mu_{1}} \sum_{s=0}^{\infty} \frac{a_{s, 1}}{z^{s}}, \quad|\operatorname{ph} z| \leqslant \frac{3}{2} \pi-\delta, \\
w_{2}(z) \sim e^{\lambda_{2} z} z^{\mu_{2}} \sum_{s=0}^{\infty}(-)^{s} \frac{a_{s, 2}}{z^{s}}, \quad-\frac{1}{2} \pi+\delta \leqslant \operatorname{ph} z \leqslant \frac{5}{2} \pi-\delta ;
\end{gathered}
$$

here and subsequently, $\delta$ denotes an arbitrary small positive constant. The exponents $\mu_{1}$ and $\mu_{2}$ are given by

$$
\begin{aligned}
& \mu_{1}=f_{1} \lambda_{1}+g_{1}, \\
& \mu_{2}=-\left(f_{1} \lambda_{2}+g_{1}\right) .
\end{aligned}
$$


The coefficients are determined by $a_{0,1}=a_{0,2}=1$ and, when $s \geqslant 1$,

$$
\begin{aligned}
-s a_{s, 1}=( & \left.s-\mu_{1}\right)\left(s-1-\mu_{1}\right) a_{s-1,1} \\
& +\sum_{j=1}^{s}\left\{\lambda_{1} f_{j+1}+g_{j+1}-\left(s-j-\mu_{1}\right) f_{j}\right\} a_{s-j, 1}, \\
-s a_{s, 2}=\left(s-\mu_{2}\right)\left(s-1-\mu_{2}\right) a_{s-1,2} & -\sum_{j=1}^{s}(-)^{j}\left\{\lambda_{2} f_{j+1}+g_{j+1}-\left(s-j-\mu_{2}\right) f_{j}\right\} a_{s-j, 2} .
\end{aligned}
$$

Other solutions of (1.1) include $w_{1}\left(z e^{-2 \pi i}\right)$ and $w_{2}\left(z e^{2 \pi i}\right)$. We assume that the coefficients $C_{1}$ and $C_{2}$ in the connection formulas

$$
\begin{aligned}
& w_{1}(z)=e^{2 \pi i \mu_{1}} w_{1}\left(z e^{-2 \pi i}\right)+C_{1} w_{2}(z), \\
& w_{2}(z)=e^{-2 \pi i \mu_{2}} w_{2}\left(z e^{2 \pi i}\right)+C_{2} w_{1}(z),
\end{aligned}
$$

are known or can be calculated (see [7]). We also assume that $C_{1}$ and $C_{2}$ are nonzero. ${ }^{1}$

The foregoing notation agrees with that of [6], except that we have replaced $a_{s, 2}$ by $(-)^{s} a_{s, 2}$. In [6], we also found it convenient to work with the functions

$$
v_{1}(z)=e^{-\lambda_{1} z} z^{-\mu_{1}-1} w_{1}(z), \quad v_{2}(z)=e^{-\lambda_{1} z} z^{-\mu_{1}-1} w_{2}(z),
$$

in place of the actual solutions $w_{1}(z)$ and $w_{2}(z)$. For reasons of symmetry, we find it more convenient in the present paper to employ the pair

$$
\begin{aligned}
& u_{1}(z)=e^{-\lambda_{1} z} z^{-\mu_{1}} w_{1}(z), \\
& u_{2}(z)=e^{-\mu_{2} \pi i} e^{\lambda_{2} z} z^{-\mu_{2}} w_{2}\left(z e^{\pi i}\right),
\end{aligned}
$$

which we shall regard as the basic functions for equation (1.1).

From (3.3) we obtain

$$
\begin{aligned}
& u_{1}(z) \sim \sum_{s=0}^{\infty} \frac{a_{s, 1}}{z^{s}}, \\
& u_{2}(z) \sim \sum_{s=0}^{\infty} \frac{a_{s, 2}}{z^{s}},
\end{aligned}
$$

each of these expansions being valid when $z \rightarrow \infty$ in $|\operatorname{ph} z| \leqslant \frac{3}{2} \pi-\delta$. In place of (3.6) we now have

$$
\begin{aligned}
& u_{1}(z)=u_{1}\left(z e^{-2 \pi i}\right)+2 \pi i K_{1} e^{-\omega \pi i} e^{z} z^{\omega} u_{2}\left(z e^{-\pi i}\right), \\
& u_{2}(z)=u_{2}\left(z e^{-2 \pi i}\right)+2 \pi i K_{2} e^{\omega \pi i} e^{z} z^{-\omega} u_{1}\left(z e^{-\pi i}\right)
\end{aligned}
$$

in which $\omega$ is defined as in (2.6) and

$$
\begin{aligned}
& K_{1}=(2 \pi i)^{-1} e^{\omega \pi i} C_{1}, \\
& K_{2}=-(2 \pi i)^{-1} C_{2} .
\end{aligned}
$$

\footnotetext{
${ }^{1}$ If, for example, $C_{1}=0$, then the expansion (3.3a) converges and can be handled quite differently; compare [6], §2, Remark (v).
} 
We shall need the following transforms of Stieltjes type:

$$
\begin{aligned}
& u_{1}(z)=K_{1} z \int_{\rho}^{\infty} \frac{e^{-t} t^{-1+\omega}}{t+z} u_{2}(t) d t-\frac{z}{2 \pi i} \int_{\rho e^{-\pi i}}^{\rho e^{\pi i}} \frac{u_{1}(t)}{t(t-z)} d t \\
& u_{2}(z)=K_{2} z \int_{\rho}^{\infty} \frac{e^{-t} t^{-1-\omega}}{t+z} u_{1}(t) d t-\frac{z}{2 \pi i} \int_{\rho e^{-\pi i}}^{\rho e^{\pi i}} \frac{u_{2}(t)}{t(t-z)} d t
\end{aligned}
$$

valid when $|z|>\rho$ and $|\operatorname{ph} z|<\pi$. Here $\rho$ is an arbitrary constant such that $\rho>a$, $a$ being defined as in $\S 1$. In each of these equations, the path in the first integral is a straight line and in the second integral is an arc of the circle $|t|=\rho$ described in the positive sense. The proof is based on equations (3.9) and is similar to the proof of Lemma 3.1 of [6]. We omit the proof.

Let $R_{1}^{(0)}\left(z ; N_{0}\right)$ and $R_{2}^{(0)}\left(z ; N_{0}\right)$ denote the remainder terms when the expansions (3.8) are terminated after $N_{0}$ terms, $N_{0}$ being an arbitrary nonnegative integer; thus

$$
\begin{aligned}
& u_{1}(z)=\sum_{s=0}^{N_{0}-1} \frac{a_{s, 1}}{z^{s}}+R_{1}^{(0)}\left(z ; N_{0}\right), \\
& u_{2}(z)=\sum_{s=0}^{N_{0}-1} \frac{a_{s, 2}}{z^{s}}+R_{2}^{(0)}\left(z ; N_{0}\right) .
\end{aligned}
$$

If we substitute into (3.11) by means of the identities

$$
\begin{aligned}
& \frac{1}{t+z}=\frac{1}{z} \sum_{s=0}^{N_{0}-1}(-)^{s}\left(\frac{t}{z}\right)^{s}+\frac{(-)^{N_{0}}}{t+z}\left(\frac{t}{z}\right)^{N_{0}}, \\
& \frac{1}{t-z}=-\frac{1}{z} \sum_{s=0}^{N_{0}-1}\left(\frac{t}{z}\right)^{s}+\frac{1}{t-z}\left(\frac{t}{z}\right)^{N_{0}},
\end{aligned}
$$

then, as in the proof of Lemma 3.2 of [6], we obtain the following integral representations

$$
\begin{aligned}
& R_{1}^{(0)}\left(z ; N_{0}\right)=(-)^{N_{0}} \frac{K_{1}}{z^{N_{0}-1}} \int_{\rho}^{\infty} \frac{e^{-t} t^{N_{0}-1+\omega}}{t+z} u_{2}(t) d t+\varepsilon_{1}\left(z ; \rho ; N_{0}\right), \\
& R_{2}^{(0)}\left(z ; N_{0}\right)=(-)^{N_{0}} \frac{K_{2}}{z^{N_{0}-1}} \int_{\rho}^{\infty} \frac{e^{-t} t^{N_{0}-1-\omega}}{t+z} u_{1}(t) d t+\varepsilon_{2}\left(z ; \rho ; N_{0}\right),
\end{aligned}
$$

when $|z|>\rho$ and $|\operatorname{ph} z|<\pi$, where

$$
\begin{aligned}
& \varepsilon_{1}\left(z ; \rho ; N_{0}\right)=-\frac{z^{1-N_{0}}}{2 \pi i} \int_{\rho e^{-\pi i}}^{\rho e^{\pi i}} \frac{t^{N_{0}-1}}{t-z} u_{1}(t) d t \\
& \varepsilon_{2}\left(z ; \rho ; N_{0}\right)=-\frac{z^{1-N_{0}}}{2 \pi i} \int_{\rho e^{-\pi i}}^{\rho e^{\pi i}} \frac{t^{N_{0}-1}}{t-z} u_{2}(t) d t
\end{aligned}
$$

We note that the functions $\varepsilon_{1}\left(z ; \rho ; N_{0}\right)$ and $\varepsilon_{2}\left(z ; \rho ; N_{0}\right)$ are analytic on the annulus $|z|>\rho$. Furthermore, if $\mathbf{A}$ denotes the closed annulus $|z| \geqslant \rho+\delta$, then

$$
\varepsilon_{1}\left(z ; \rho ; N_{0}\right), \varepsilon_{2}\left(z ; \rho ; N_{0}\right)=\mathcal{O}\left(\rho^{N_{0}} z^{-N_{0}}\right),
$$

where the $\mathcal{O}$-term is uniform with respect to $z \in \mathbf{A}$ and all nonnegative integers $N_{0}$. 


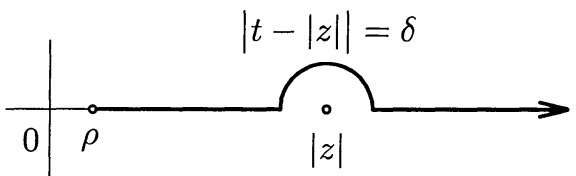

Figure 3.1. $t$-plane. Path $\mathcal{P}$ when $|z| \geqslant \rho+\delta$ and $0 \leqslant \mathrm{ph} z \leqslant \pi$.

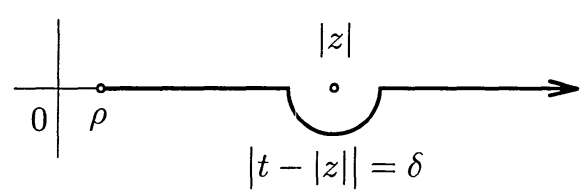

Figure 3.2. $t$-plane. Path $\mathcal{P}$ when $|z| \geqslant \rho+\delta$ and $-\pi \leqslant \operatorname{ph} z<0$.

We further note that the process of substituting into (3.11) by means of (3.13) also yields integral representations for the coefficients $a_{s, 1}$ and $a_{s, 2}$. From these representations asymptotic expansions of $a_{s, 1}$ and $a_{s, 2}$ for large $s$ can be constructed in inverse factorial series. This result appears as Theorem 2.1 in [6], and for later reference we record these expansions in the present notation:

$$
\begin{aligned}
& a_{s, 1} \sim(-)^{s} K_{1} \sum_{j=0}^{\infty} a_{j, 2} \Gamma(s-j+\omega), \\
& a_{s, 2} \sim(-)^{s} K_{2} \sum_{j=0}^{\infty} a_{j, 1} \Gamma(s-j-\omega) .
\end{aligned}
$$

The final step in this section is to modify the representations (3.14) in such a way that they include the phases $\mathrm{ph} z= \pm \pi$. This is achieved by restricting $z \in \mathbf{A}$ and deforming the integration path by a semicircular indentation of radius $\delta$ that is centered at $|z|$, and passes above $|z|$ when $0 \leqslant \operatorname{ph} z \leqslant \pi$ and below $|z|$ when $-\pi \leqslant \operatorname{ph} z<0$; see Figures 3.1 and 3.2. We denote the deformed path by $\mathcal{P}$. Then by continuity we derive

$$
\begin{aligned}
& R_{1}^{(0)}\left(z ; N_{0}\right)=(-)^{N_{0}} \frac{K_{1}}{z^{N_{0}-1}} \int_{\mathcal{P}} \frac{e^{-t} t^{N_{0}-1+\omega}}{t+z} u_{2}(t) d t+\varepsilon_{1}\left(z ; \rho ; N_{0}\right), \\
& R_{2}^{(0)}\left(z ; N_{0}\right)=(-)^{N_{0}} \frac{K_{2}}{z^{N_{0}-1}} \int_{\mathcal{P}} \frac{e^{-t} t^{N_{0}-1-\omega}}{t+z} u_{1}(t) d t+\varepsilon_{2}\left(z ; \rho ; N_{0}\right),
\end{aligned}
$$

valid when $z \in \mathbf{A}$ and $|\operatorname{ph} z| \leqslant \pi$.

\section{Optimal expansions at level zero (Poincaré's expansions)}

Throughout this section, we suppose that $|\operatorname{ph} z| \leqslant \pi$.

We seek to estimate and then optimize the remainder terms $R_{1}^{(0)}\left(z ; N_{0}\right)$ and $R_{2}^{(0)}\left(z ; N_{0}\right)$ that appear in $(3.12)$ by permitting $N_{0}$ to be a linear function of $|z|$. More precisely, we assume that

$$
N_{0}=\beta_{0}|z|+\alpha_{0}
$$

where $\beta_{0}$ is a positive constant at our disposal and $\alpha_{0}$ is bounded. ${ }^{2}$ Whatever the choice of $\beta_{0}$ and $\alpha_{0}$, we assume throughout that $|z|$ is restricted in such a way that

$$
N_{0} \geqslant 1+|\Re \omega| \text {. }
$$

\footnotetext{
${ }^{2}$ Typically, $N_{0}=\operatorname{int}\left[\beta_{0}|z|\right]$, so that $-1<\alpha_{0} \leqslant 0$.
} 
This is obviously achieved if we restrict $z \in \mathbf{A}_{0}$, where $\mathbf{A}_{0}$ is the closed annulus defined by

$$
|z| \geqslant\left(1+|\Re \omega|-\tilde{\alpha}_{0}\right) / \beta_{0},
$$

$\tilde{\alpha}_{0}$ being the lesser of $1+|\Re \omega|$ and $\lim \inf \alpha_{0}$.

In the representation (3.18a), we observe that because $z \in \mathbf{A}$, we have $|t+z| \geqslant \delta$ everywhere on $\mathcal{P}$. Since also $u_{2}(t)=\mathcal{O}(1)$ as $t \rightarrow \infty$, it follows that the contribution of the straight-line segments of $\mathcal{P}$ is estimated by

$$
\left(\int_{\rho}^{|z|-\delta}+\int_{|z|+\delta}^{\infty}\right) \frac{e^{-t} t^{N_{0}-1+\omega}}{t+z} u_{2}(t) d t=\Gamma\left(N_{0}+\Re \omega\right) \mathcal{O}(1),
$$

uniformly with respect to $z \in \mathbf{A} \cap \mathbf{A}_{0}$. On the semicircular arc we have

$$
\int_{|z|-\delta}^{|z|+\delta} \frac{e^{-t} t^{N_{0}-1+\omega}}{t+z} u_{2}(t) d t=\mathcal{O}\left\{e^{-|z|}(|z|+\delta)^{N_{0}-1+\Re \omega}\right\}
$$

also uniformly on $\mathbf{A} \cap \mathbf{A}_{0}$. On referring to (4.1) and applying Stirling's formula, we see that

$$
\frac{e^{-|z|}(|z|+\delta)^{N_{0}-1+\Re \omega}}{\Gamma\left(N_{0}+\Re \omega\right)}=e^{-|z|}\left(\beta_{0}^{\beta_{0}} e^{-\beta_{0}}\right)^{-|z|} z^{-\frac{1}{2}} \mathcal{O}(1)=\mathcal{O}\left(z^{-\frac{1}{2}}\right)
$$

uniformly on $\mathbf{A} \cap \mathbf{A}_{0}$. The last step follows from the observation that $\beta_{0}^{\beta_{0}} e^{-\beta_{0}} \geqslant e^{-1}$ for all positive values of $\beta_{0}$. Accordingly, on combining (4.4) and (4.5) we have

$$
(-)^{N_{0}} \frac{K_{1}}{z^{N_{0}-1}} \int_{\mathcal{P}} \frac{e^{-t} t^{N_{0}-1+\omega}}{t+z} u_{2}(t) d t=\Gamma\left(N_{0}+\Re \omega\right) \mathcal{O}\left(z^{1-N_{0}}\right),
$$

uniformly on $\mathbf{A} \cap \mathbf{A}_{0}$.

The other contribution to $R_{1}^{(0)}\left(z ; N_{0}\right)$ in (3.18a), namely $\varepsilon_{1}\left(z ; \rho ; N_{0}\right)$, is estimated by (3.16). Obviously, this contribution, too, may be absorbed into the right-hand side of (4.6). We therefore have proved that

$$
R_{1}^{(0)}\left(z ; N_{0}\right)=\Gamma\left(N_{0}+\Re \omega\right) \mathcal{O}\left(z^{1-N_{0}}\right)
$$

and, hence,

$$
R_{1}^{(0)}\left(z ; N_{0}\right)=\left(\beta_{0}^{\beta_{0}} e^{-\beta_{0}}\right)^{|z|} \mathcal{O}\left(z^{\frac{1}{2}+\Re \omega}\right),
$$

uniformly on $\mathbf{A} \cap \mathbf{A}_{0}$ in both instances. And, in the same circumstances, we have, by symmetry,

$$
R_{2}^{(0)}\left(z ; N_{0}\right)=\Gamma\left(N_{0}-\Re \omega\right) \mathcal{O}\left(z^{1-N_{0}}\right)=\left(\beta_{0}^{\beta_{0}} e^{-\beta_{0}}\right)^{|z|} \mathcal{O}\left(z^{\frac{1}{2}-\Re \omega}\right) .
$$

The estimates (4.8) and (4.9) apply for any value of the positive constant $\beta_{0}$, but they are minimal when $\beta_{0}=1$. Then letting $z \rightarrow \infty$ we arrive at the main result of this section: if $z \rightarrow \infty$ in $|\mathrm{ph} z| \leqslant \pi$ and $N_{0}=|z|+\mathcal{O}(1)$, then

$$
\begin{aligned}
& R_{1}^{(0)}\left(z ; N_{0}\right)=\mathcal{O}\left(e^{-|z|} z^{\frac{1}{2}+\Re \omega}\right), \\
& R_{2}^{(0)}\left(z ; N_{0}\right)=\mathcal{O}\left(e^{-|z|} z^{\frac{1}{2}-\Re \omega}\right) .
\end{aligned}
$$


Remark. The estimates (4.10) can also be obtained by setting $m=0$ in Theorem 2.2 of [6]. Indeed, in this way we find that they can be improved to

$$
\begin{aligned}
& R_{1}^{(0)}\left(z ; N_{0}\right)=\mathcal{O}\left(e^{-|z|} z^{\Re \omega}\right), \\
& R_{2}^{(0)}\left(z ; N_{0}\right)=\mathcal{O}\left(e^{-|z|} z^{-\Re \omega}\right) .
\end{aligned}
$$

We have included the analysis of this section for the following reasons. First, we wanted to show that $\beta_{0}=1$ is optimal by analysis of the remainder terms instead of the more usual analysis of the actual terms. Secondly, we need some of the intermediate results later. Thirdly, with a method similar to the proof of Lemma 2.1, with $l=2$, we can obtain (4.11) directly from the integral representations (3.18).

\section{Level one (exponentially-improved expansions)}

Throughout this section, we suppose that $|\operatorname{ph} z| \leqslant \pi$ and that $\rho$ and $\delta$ are chosen to satisfy $\rho-\delta>a$.

In (3.12b), replace $z$ by $t$ and $N_{0}$ by another arbitrary nonnegative integer $N_{1}$. On substituting the result into $(3.18 a)$, we obtain

$$
\begin{aligned}
R_{1}^{(0)}(z ; & \left.N_{0}\right)=(-)^{N_{0}} \frac{K_{1}}{z^{N_{0}-1}} \sum_{s=0}^{N_{1}-1} a_{s, 2} \int_{\mathcal{P}} \frac{e^{-t} t^{N_{0}-s-1+\omega}}{t+z} d t \\
& +(-)^{N_{0}} \frac{K_{1}}{z^{N_{0}-1}} \int_{\mathcal{P}} \frac{e^{-t} t^{N_{0}-1+\omega}}{t+z} R_{2}^{(0)}\left(t ; N_{1}\right) d t+\varepsilon_{1}\left(z ; \rho ; N_{0}\right),
\end{aligned}
$$

valid when $z \in \mathbf{A}$. To ensure convergence of all integrals that appear below, we restrict the choice of $N_{0}$ and $N_{1}$ by condition (4.2) and also

$$
N_{1}, N_{0}-N_{1} \geqslant 1+|\Re \omega| \text {. }
$$

When $z \in \mathbf{A}$, we may deform the integration path in $(2.2 \mathrm{~b})$ into the union of $\mathcal{P}$ and the interval $[0, \rho]$ (thereby making $(2.2 b)$ valid when $|\mathrm{ph} z| \leqslant \pi$ ). On substituting into (5.1) by means of this result, we obtain

$$
R_{1}^{(0)}\left(z ; N_{0}\right)=(-)^{N_{0}} \frac{K_{1}}{z^{N_{0}-1}} \sum_{s=0}^{N_{1}-1} a_{s, 2} G_{\omega}^{(1)}\left(z ; N_{0}-s\right)+R_{1}^{(1)}\left(z ; N_{0}, N_{1}\right),
$$

where

$$
\begin{aligned}
& R_{1}^{(1)}\left(z ; N_{0},\right.\left.N_{1}\right)=(-)^{N_{0}-1} \frac{K_{1}}{z^{N_{0}-1}} \sum_{s=0}^{N_{1}-1} a_{s, 2} \int_{0}^{\rho} \frac{e^{-t} t^{N_{0}-s-1+\omega}}{t+z} d t \\
&+(-)^{N_{0}} \frac{K_{1}}{z^{N_{0}-1}} \int_{\mathcal{P}} \frac{e^{-t} t^{N_{0}-1+\omega}}{t+z} R_{2}^{(0)}\left(t ; N_{1}\right) d t+\varepsilon_{1}\left(z ; \rho ; N_{0}\right),
\end{aligned}
$$

again valid when $z \in \mathbf{A}$.

Equation (5.3) is the desired re-expansion of $R_{1}^{(0)}\left(z ; N_{0}\right)$. We now seek to optimize the new remainder term $R_{1}^{(1)}\left(z ; N_{0}, N_{1}\right)$ by assuming that (4.1) applies and also that

$$
N_{1}=\beta_{1}|z|+\alpha_{1},
$$

where $\beta_{1} \in\left(0, \beta_{0}\right)$ is another constant at our disposal and $\alpha_{1}$ is bounded. The conditions (4.2) and (5.2) are satisfied when $z \in \mathbf{A}_{1}$, where $\mathbf{A}_{1}$ is an unbounded closed 
annulus centered at the origin and of sufficiently large radius; compare (4.3). Obviously, $\mathbf{A}_{1} \subseteq \mathbf{A}_{0}$.

We first observe that we cannot use the uniform estimates $(4.9)$ for $R_{2}^{(0)}\left(z ; N_{0}\right)$ to estimate the integral along $\mathcal{P}$ on the right-hand side of (5.4). This is because (4.9), with $z=t$ and $N_{0}=N_{1}$, does not apply to all values of $t$ on $\mathcal{P}$. Instead, we substitute into this integral by means of (3.14b), with $z, \rho, N_{0}$, and $t$ replaced by $t, \rho-\delta, N_{1}$, and $t_{1}$, respectively. This yields

$$
\begin{aligned}
R_{1}^{(1)}\left(z ; N_{0}, N_{1}\right)= & (-)^{N_{0}+N_{1}} \frac{K_{1} K_{2}}{z^{N_{0}-1}} \int_{\mathcal{P}} \int_{\rho-\delta}^{\infty} \frac{e^{-t} t^{N_{0}-N_{1}+\omega}}{t+z} \frac{e^{-t_{1}} t_{1}^{N_{1}-1-\omega}}{t_{1}+t} u_{1}\left(t_{1}\right) d t_{1} d t \\
& +S_{1}^{(1)}\left(z ; N_{0}, N_{1}\right)
\end{aligned}
$$

where

$$
\begin{aligned}
S_{1}^{(1)}\left(z ; N_{0}, N_{1}\right)= & (-)^{N_{0}-1} \frac{K_{1}}{z^{N_{0}-1}} \sum_{s=0}^{N_{1}-1} a_{s, 2} \int_{0}^{\rho} \frac{e^{-t} t^{N_{0}-s-1+\omega}}{t+z} d t \\
& +(-)^{N_{0}} \frac{K_{1}}{z^{N_{0}-1}} \int_{\mathcal{P}} \frac{e^{-t} t^{N_{0}-1+\omega}}{t+z} \varepsilon_{2}\left(t ; \rho-\delta ; N_{1}\right) d t+\varepsilon_{1}\left(z ; \rho ; N_{0}\right),
\end{aligned}
$$

again valid when $z \in \mathbf{A}_{1}$.

We first estimate $S_{1}^{(1)}\left(z ; N_{0}, N_{1}\right)$. To begin with, we have from (3.17b)

$$
a_{s, 2}=N_{1}^{-1} \Gamma\left(N_{1}-\Re \omega\right) \mathcal{O}(1)
$$

uniformly for $s=0,1, \ldots, N_{1}-1$ and all integers $N_{1}$ that satisfy (5.2a). Next, since $N_{0}-N_{1}+\Re \omega \leqslant N_{0}-s-1+\Re \omega \leqslant N_{0}-1+\Re \omega$, it follows that

$$
\int_{0}^{\rho} \frac{e^{-t} t^{N_{0}-s-1+\omega}}{t+z} d t=\mathcal{O}\left(\rho_{1}^{N_{0}} z^{-1}\right)
$$

uniformly for $s=0,1, \ldots, N_{1}-1$ and $z \in \mathbf{A} \cap \mathbf{A}_{1}$, where $\rho_{1}=\max (\rho, 1)$. Hence, for the sum on the right-hand side of (5.7), we have

$$
(-)^{N_{0}-1} \frac{K_{1}}{z^{N_{0}-1}} \sum_{s=0}^{N_{1}-1} a_{s, 2} \int_{0}^{\rho} \frac{e^{-t} t^{N_{0}-s-1+\omega}}{t+z} d t=\rho_{1}^{N_{0}} z^{-N_{0}} \Gamma\left(N_{1}-\Re \omega\right) \mathcal{O}(1),
$$

uniformly on $\mathbf{A} \cap \mathbf{A}_{1}$.

For the second term on the right-hand side of $(5.7)$, we note that from $(3.16 \mathrm{~b})$

$$
\varepsilon_{2}\left(t ; \rho-\delta ; N_{1}\right)=(\rho-\delta)^{N_{1}} t^{-N_{1}} \mathcal{O}(1) \subseteq \mathcal{O}\left(\rho^{N_{1}} t^{-N_{1}}\right)
$$

uniformly for $t$ in the annulus $|t| \geqslant \rho$ and all nonnegative integers $N_{1}$. Then, by use of analysis similar to that used to establish (4.6), we obtain

$(-)^{N_{0}} \frac{K_{1}}{z^{N_{0}-1}} \int_{\mathcal{P}} \frac{e^{-t} t^{N_{0}-1+\omega}}{t+z} \varepsilon_{2}\left(t ; \rho-\delta ; N_{1}\right) d t=\rho^{N_{1}} z^{1-N_{0}} \Gamma\left(N_{0}-N_{1}+\Re \omega\right) \mathcal{O}(1)$,

uniformly on $\mathbf{A} \cap \mathbf{A}_{1}$. 
The remaining term in (5.7) is estimated directly by (3.16a). In consequence, it may be absorbed into the right-hand side of (5.9). We may combine the foregoing results into the following form:

$$
S_{1}^{(1)}\left(z ; N_{0}, N_{1}\right)=\left\{\rho_{1}^{N_{0}} \Gamma\left(N_{1}-\Re \omega\right)+\rho^{N_{1}} \Gamma\left(N_{0}-N_{1}+\Re \omega\right)\right\} \mathcal{O}\left(z^{1-N_{0}}\right),
$$

uniformly on $\mathbf{A} \cap \mathbf{A}_{1}$.

We now turn our attention to the double integral in (5.6). In the inner integral, we have $\left|t_{1}+t\right| \geqslant|t|$ when $t \in \mathcal{P}$; also $u_{1}\left(t_{1}\right)=\mathcal{O}(1)$. Hence,

$$
\int_{\rho-\delta}^{\infty} \frac{e^{-t_{1}} t_{1}^{N_{1}-1-\omega}}{t_{1}+t} u_{1}\left(t_{1}\right) d t_{1}=t^{-1} \Gamma\left(N_{1}-\Re \omega\right) \mathcal{O}(1)
$$

uniformly for $t \in \mathcal{P}$. Accordingly, we have

$$
\begin{gathered}
(-)^{N_{0}+N_{1}} \frac{K_{1} K_{2}}{z^{N_{0}-1}} \int_{\mathcal{P}} \int_{\rho-\delta}^{\infty} \frac{e^{-t} t^{N_{0}-N_{1}+\omega}}{t+z} \frac{e^{-t_{1}} t_{1}^{N_{1}-1-\omega}}{t_{1}+t} u_{1}\left(t_{1}\right) d t_{1} d t \\
=\Gamma\left(N_{0}-N_{1}+\Re \omega\right) \Gamma\left(N_{1}-\Re \omega\right) \mathcal{O}\left(z^{1-N_{0}}\right),
\end{gathered}
$$

uniformly on $\mathbf{A} \cap \mathbf{A}_{1}$; compare again the derivation of (4.6).

Let us now compare (5.12) with (5.13). It easily is seen that as a consequence of (4.1), (5.2), and (5.5), each of the ratios

$$
\frac{\rho_{1}^{N_{0}}}{\Gamma\left(N_{0}-N_{1}+\Re \omega\right)}, \quad \frac{\rho^{N_{1}}}{\Gamma\left(N_{1}-\Re \omega\right)}
$$

is bounded on $\mathbf{A} \cap \mathbf{A}_{1}$. In consequence, the estimate (5.12) for $S_{1}^{(1)}\left(z ; N_{0}, N_{1}\right)$ may be absorbed into (5.13). We therefore have proved that

$$
R_{1}^{(1)}\left(z ; N_{0}, N_{1}\right)=\Gamma\left(N_{0}-N_{1}+\Re \omega\right) \Gamma\left(N_{1}-\Re \omega\right) \mathcal{O}\left(z^{1-N_{0}}\right)
$$

uniformly on $\mathbf{A} \cap \mathbf{A}_{1}$.

If we substitute into (5.15) by means of (4.1) and (5.5) and let $z \rightarrow \infty$, then with the aid once again of Stirling's formula, we find that

$$
R_{1}^{(1)}\left(z ; N_{0}, N_{1}\right)=\left\{\left(\beta_{0}-\beta_{1}\right)^{\beta_{0}-\beta_{1}} e^{-\left(\beta_{0}-\beta_{1}\right)}\right\}^{|z|}\left(\beta_{1}^{\beta_{1}} e^{-\beta_{1}}\right)^{|z|} \mathcal{O}(1) .
$$

This estimate is minimal when $\beta_{0}-\beta_{1}=1$ and $\beta_{1}=1$, that is, when $N_{0}=2|z|+\mathcal{O}(1)$ and $N_{1}=|z|+\mathcal{O}(1)$, and it becomes

$$
R_{1}^{(1)}\left(z ; N_{0}, N_{1}\right)=\mathcal{O}\left(e^{-2|z|}\right)
$$

By analysis similar to the proof of Lemma 2.1, we can sharpen the estimate (5.17) by a factor $z^{-\frac{1}{2}}$, as follows. When $|\operatorname{ph} z| \leqslant \pi-\delta$, we may use the inequality $|t+z| \geqslant|z| \sin \delta$ in place of $|t+z| \geqslant \delta$ in (5.13) to replace the term $\mathcal{O}\left(z^{1-N_{0}}\right)$ on the right-hand side by $\mathcal{O}\left(z^{-N_{0}}\right)$. When $\delta \leqslant \pm \operatorname{ph} z \leqslant \pi$, we substitute by means of (2.14) and the corresponding 
form of (2.14) when $e^{-\pi i} z$ is replaced by $e^{\pi i} z$. This yields

$$
\begin{aligned}
\int_{\mathcal{P}} \int_{\rho-\delta}^{\infty} & \frac{e^{-t} t^{N_{0}-N_{1}+\omega}}{t+z} \frac{e^{-t_{1}} t_{1}^{N_{1}-1-\omega}}{t_{1}+t} u_{1}\left(t_{1}\right) d t_{1} d t \\
= & G_{\omega}^{(1)}\left(z ; N_{0}-N_{1}+1\right) \int_{\rho-\delta}^{\infty} \frac{e^{-t_{1}} t_{1}^{N_{1}-1-\omega}}{t_{1}+e^{\mp \pi i} z} u_{1}\left(t_{1}\right) d t_{1} \\
& -\int_{0}^{\rho} \int_{\rho-\delta}^{\infty} \frac{e^{-t} t^{N_{0}-N_{1}+\omega}}{t+z} \frac{e^{-t_{1}} t_{1}^{N_{1}-1-\omega}}{t_{1}+e^{\mp \pi i} z} u_{1}\left(t_{1}\right) d t_{1} d t \\
& -\int_{\mathcal{P}} \int_{\rho-\delta}^{\infty} \frac{e^{-t} t^{N_{0}-N_{1}+\omega}}{t_{1}+e^{\mp \pi i} z} \frac{e^{-t_{1}} t_{1}^{N_{1}-1-\omega}}{t_{1}+t} u_{1}\left(t_{1}\right) d t_{1} d t
\end{aligned}
$$

The first term on the right-hand side can be estimated with the aid of (2.13). Moreover, the resulting estimate for this term is found to dominate those for the second and third terms. Thus, we have

$$
\begin{array}{r}
(-)^{N_{0}+N_{1}} \frac{K_{1} K_{2}}{z^{N_{0}-1}} \int_{\mathcal{P}} \int_{\rho-\delta}^{\infty} \frac{e^{-t} t^{N_{0}-N_{1}+\omega}}{t+z} \frac{e^{-t_{1}} t_{1}^{N_{1}-1-\omega}}{t_{1}+t} u_{1}\left(t_{1}\right) d t_{1} d t \\
=\Gamma\left(N_{0}-N_{1}+\Re \omega\right) \Gamma\left(N_{1}-\Re \omega\right) \mathcal{O}\left(z^{\frac{1}{2}-N_{0}}\right),
\end{array}
$$

when $\delta \leqslant \pm \operatorname{ph} z \leqslant \pi$ and, hence, also when $|\operatorname{ph} z| \leqslant \pi$; compare the second sentence of this paragraph. The other term in (5.6), namely $S_{1}^{(1)}\left(z ; N_{0}, N_{1}\right)$, is absorbable in this new estimate because the ratios (5.14) are certainly $\mathcal{O}\left(z^{-\frac{1}{2}}\right)$ as $z \rightarrow \infty$. Accordingly, we arrive at the main result of this section: if $z \rightarrow \infty$ in $|\mathrm{ph} z| \leqslant \pi$ and $N_{0}=2|z|+\mathcal{O}(1)$, $N_{1}=|z|+\mathcal{O}(1)$, then

$$
R_{1}^{(1)}\left(z ; N_{0}, N_{1}\right)=\mathcal{O}\left(e^{-2|z|} z^{-\frac{1}{2}}\right)
$$

By symmetry, in the same circumstances, we have

$$
R_{2}^{(0)}\left(z ; N_{0}\right)=(-)^{N_{0}} \frac{K_{2}}{z^{N_{0}-1}} \sum_{s=0}^{N_{1}-1} a_{s, 1} G_{-\omega}^{(1)}\left(z ; N_{0}-s\right)+R_{2}^{(1)}\left(z ; N_{0}, N_{1}\right)
$$

where

$$
R_{2}^{(1)}\left(z ; N_{0}, N_{1}\right)=\mathcal{O}\left(e^{-2|z|} z^{-\frac{1}{2}}\right) .
$$

Remark (i). This result is similar to Theorem 2.2 of [6]. However, there is a significant difference. In [6], we truncated each of the expansions (3.12) at (or near) its smallest term and then re-expanded the remainder term in the form (5.3). In the present notation, this sets $\beta_{0}=1$. With this constraint, the error terms $R_{1}^{(1)}\left(z ; N_{0}, N_{1}\right)$ and $R_{2}^{(1)}\left(z ; N_{0}, N_{1}\right)$ are optimized when $\beta_{1}=\frac{1}{2} ;$ compare (5.16). In full, we have $N_{0}=|z|+\mathcal{O}(1), N_{1}=\frac{1}{2}|z|+\mathcal{O}(1)$ and

$$
R_{1}^{(1)}\left(z ; N_{0}, N_{1}\right), \quad R_{2}^{(1)}\left(z ; N_{0}, N_{1}\right)=\mathcal{O}\left(2^{-|z|} e^{-|z|}\right) .
$$

As expected, these estimates are weaker than (5.19) and (5.21). In other words, it is more accurate to continue the expansions (3.12) beyond their smallest terms, in fact, to take as many terms again and then re-expand the remainder in series of $G_{ \pm \omega}^{(1)}$ 
functions (or equivalently $F_{p}$ functions) until we reach the vicinity of the smallest term of the re-expanded series.

Remark (ii). It should be noted that in sharpening the estimate (5.17) into (5.19), we needed the condition $N_{0}-N_{1}=|z|+\mathcal{O}(1)$ in order to be able to estimate $G_{\omega}^{(1)}\left(z ; N_{0}-N_{1}+1\right)$ by means of (2.13). To put this another way, the sharpening does not become available until the original estimate (5.16) has been optimized.

\section{Level two}

Throughout this section, we suppose that $|\operatorname{ph} z| \leqslant \pi$ and $\rho-2 \delta>a$.

Our purpose is to re-expand $R_{1}^{(1)}\left(z ; N_{0}, N_{1}\right)$ and $R_{2}^{(1)}\left(z ; N_{0}, N_{1}\right)$, and we proceed in a manner analogous to the two preceding sections. We substitute into (5.6) by means of (3.12a) and (3.14a), with $z, \rho, N_{0}$, and $t$ replaced by $t_{1}, \rho-2 \delta, N_{2}$, and $t_{2}$, respectively, and refer to $(2.2 \mathrm{c})$ with $l=2, M_{0}=N_{0}-N_{1}, M_{1}=N_{1}-s$. Again, $N_{0}$, $N_{1}, N_{2}$ are arbitrary integers. These substitutions are certainly valid when (4.2) and (5.2) apply, and also

$$
N_{2}, N_{1}-N_{2} \geqslant 1+|\Re \omega|
$$

We arrive at

$$
\begin{aligned}
R_{1}^{(1)}\left(z ; N_{0}, N_{1}\right)=( & -)^{N_{0}+N_{1}} \frac{K_{1} K_{2}}{z^{N_{0}-1}} \sum_{s=0}^{N_{2}-1} a_{s, 1} G_{\omega}^{(2)}\left(z ; N_{0}-N_{1}, N_{1}-s\right) \\
& +R_{1}^{(2)}\left(z ; N_{0}, N_{1}, N_{2}\right)
\end{aligned}
$$

where

$$
\begin{aligned}
& R_{1}^{(2)}\left(z ; N_{0}, N_{1}, N_{2}\right)=(-)^{N_{0}+N_{1}+N_{2}} \frac{K_{1}^{2} K_{2}}{z^{N_{0}-1}} \times \\
& \quad \times \int_{\mathcal{P}} \int_{\rho-\delta}^{\infty} \int_{\rho-2 \delta}^{\infty} \frac{e^{-t-t_{1}-t_{2}} t^{N_{0}-N_{1}+\omega_{1}} t_{1}^{N_{1}-N_{2}-\omega} t_{2}^{N_{2}-1+\omega}}{(t+z)\left(t_{1}+t\right)\left(t_{2}+t_{1}\right)} u_{2}\left(t_{2}\right) d t_{2} d t_{1} d t \\
& +S_{1}^{(2)}\left(z ; N_{0}, N_{1}, N_{2}\right)
\end{aligned}
$$

and

$$
\begin{aligned}
S_{1}^{(2)}(z ; & \left.N_{0}, N_{1}, N_{2}\right)=(-)^{N_{0}+N_{1}-1} \frac{K_{1} K_{2}}{z^{N_{0}-1}} \times \\
& \times \sum_{s=0}^{N_{2}-1} a_{s, 1}\left(\int_{0}^{\rho} \int_{0}^{\infty}+\int_{\mathcal{P}} \int_{0}^{\rho-\delta}\right) \frac{e^{-t-t_{1}} t^{N_{0}-N_{1}+\omega_{1}} t_{1}^{N_{1}-s-1-\omega}}{(t+z)\left(t_{1}+t\right)} d t_{1} d t \\
+ & (-)^{N_{0}+N_{1}} \frac{K_{1} K_{2}}{z^{N_{0}-1}} \int_{\mathcal{P}} \int_{\rho-\delta}^{\infty} \frac{e^{-t-t_{1}} t^{N_{0}-N_{1}+\omega} t_{1}^{N_{1}-1-\omega}}{(t+z)\left(t_{1}+t\right)} \varepsilon_{1}\left(t_{1} ; \rho-2 \delta ; N_{2}\right) d t_{1} d t \\
+ & S_{1}^{(1)}\left(z ; N_{0}, N_{1}\right)
\end{aligned}
$$

valid when $z \in \mathbf{A}$; compare (5.6) and (5.7).

Again, following the analysis of $\S \S 4$ and 5, we seek to estimate, and then optimize, the new remainder term $R_{1}^{(2)}\left(z ; N_{0}, N_{1}, N_{2}\right)$, by setting

$$
N_{0}=\beta_{0}|z|+\alpha_{0}, \quad N_{1}=\beta_{1}|z|+\alpha_{1}, \quad N_{2}=\beta_{2}|z|+\alpha_{2},
$$


where $\beta_{0}, \beta_{1}, \beta_{2}$ are constants such that $0<\beta_{2}<\beta_{1}<\beta_{0}$ and $\alpha_{0}, \alpha_{1}, \alpha_{2}$ are bounded. We restrict $z \in \mathbf{A}_{2}$, where $\mathbf{A}_{2}$ is an unbounded closed annulus centered at the origin and of radius sufficiently large to ensure that the conditions (4.2), (5.2), and (6.1) are fulfilled. Thus, $\mathbf{A}_{2} \subseteq \mathbf{A}_{1} \subseteq \mathbf{A}_{0}$.

We first observe that

$$
\begin{aligned}
a_{s, 1} \int_{0}^{\rho} \int_{0}^{\infty} \frac{e^{-t-t_{1}} t^{N_{0}-N_{1}+\omega} t_{1}^{N_{1}-s-1-\omega}}{(t+z)\left(t_{1}+t\right)} d t_{1} d t \\
=\Gamma\left(s_{1}\right) \Gamma\left(N_{1}-s-1-\Re \omega\right) \rho^{N_{0}-N_{1}} \mathcal{O}(1),
\end{aligned}
$$

where $s_{1}=\max (s+\Re \omega, 1)$, uniformly for $z \in \mathbf{A} \cap \mathbf{A}_{2}$ and $s=0,1, \ldots, N_{2}-1$. If $s+\Re \omega<1$, then

$$
\Gamma\left(s_{1}\right) \Gamma\left(N_{1}-s-1-\Re \omega\right) \leqslant \Gamma\left(N_{1}-1-\Re \omega\right),
$$

whereas if $s+\Re \omega \geqslant 1$, then ${ }^{3}$

$$
\Gamma\left(s_{1}\right) \Gamma\left(N_{1}-s-1-\Re \omega\right) \leqslant \Gamma\left(N_{1}-1\right) .
$$

With the aid, also, of (6.1b), we conclude that

$$
\sum_{s=0}^{N_{2}-1} a_{s, 1} \int_{0}^{\rho} \int_{0}^{\infty} \frac{e^{-t-t_{1}} t^{N_{0}-N_{1}+\omega_{1}} t_{1}^{N_{1}-s-1-\omega}}{(t+z)\left(t_{1}+t\right)} d t_{1} d t=\rho^{N_{0}-N_{1}} \Gamma\left(N_{1}+|\Re \omega|\right) \mathcal{O}(1)
$$

uniformly on $\mathbf{A} \cap \mathbf{A}_{2}$. The rest of the analysis is similar to that of $\S 5$, and there is no need to enter into all the details. Corresponding to (5.12) and (5.15), we find that

$$
\begin{gathered}
S_{1}^{(2)}\left(z ; N_{0}, N_{1}, N_{2}\right)=\left\{\rho^{N_{0}-N_{1}} \Gamma\left(N_{1}+|\Re \omega|\right)+\rho_{1}^{N_{1}} \Gamma\left(N_{0}-N_{1}+\Re \omega\right) \Gamma\left(N_{2}+\Re \omega\right)\right. \\
\left.+\rho^{N_{2}} \Gamma\left(N_{0}-N_{1}+\Re \omega\right) \Gamma\left(N_{1}-N_{2}-\Re \omega\right)\right\} \mathcal{O}\left(z^{1-N_{0}}\right)+S_{1}^{(1)}\left(z ; N_{0}, N_{1}\right),
\end{gathered}
$$

and

$$
\begin{aligned}
& R_{1}^{(2)}\left(z ; N_{0}, N_{1}, N_{2}\right) \\
& \quad=\Gamma\left(N_{0}-N_{1}+\Re \omega\right) \Gamma\left(N_{1}-N_{2}-\Re \omega\right) \Gamma\left(N_{2}+\Re \omega\right) \mathcal{O}\left(z^{1-N_{0}}\right),
\end{aligned}
$$

uniformly on $\mathbf{A} \cap \mathbf{A}_{2}$ in both equations.

On substituting into the last result by means of (6.5), we obtain

$$
\begin{aligned}
R_{1}^{(2)}(z ; & \left.N_{0}, N_{1}, N_{2}\right)=\left\{\left(\beta_{0}-\beta_{1}\right)^{\beta_{0}-\beta_{1}} e^{\beta_{1}-\beta_{0}}\right\}^{|z|} \times \\
& \times\left\{\left(\beta_{1}-\beta_{2}\right)^{\beta_{1}-\beta_{2}} e^{\beta_{2}-\beta_{1}}\right\}^{|z|}\left(\beta_{2}^{\beta_{2}} e^{-\beta_{2}}\right)^{|z|} \mathcal{O}\left(z^{-\frac{1}{2}+\Re \omega}\right),
\end{aligned}
$$

uniformly on $\mathbf{A} \cap \mathbf{A}_{2}$ and, in particular, as $z \rightarrow \infty$. The minimizing choice is given by $\beta_{0}-\beta_{1}=\beta_{1}-\beta_{2}=\beta_{2}=1$, that is, $\beta_{0}=3, \beta_{1}=2, \beta_{2}=1$. The corresponding estimate for $R_{2}^{(2)}\left(z ; N_{0}, N_{1}, N_{2}\right)$ is obtained by replacing $\Re \omega$ by $-\Re \omega$. Again, with

\footnotetext{
${ }^{3}$ In deriving (6.7b), we have used the inequality $\Gamma(x) \Gamma(y) \leqslant \Gamma(x+y)$ when $x, y \geqslant 1$; see, e.g., [8, Chapter $2, \S 1.6]$.
} 
these choices for $\beta_{0}, \beta_{1}, \beta_{2}$, we can sharpen the minimal estimates with a factor $z^{-\frac{1}{2}}$; compare (5.17) and (5.19). Thus we have the main result of this section: if $z \rightarrow \infty$ in $|\operatorname{ph} z| \leqslant \pi$ and $N_{0}=3|z|+\mathcal{O}(1), N_{1}=2|z|+\mathcal{O}(1), N_{2}=|z|+\mathcal{O}(1)$, then

$$
R_{1}^{(2)}\left(z ; N_{0}, N_{1}, N_{2}\right)=\mathcal{O}\left(e^{-3|z|} z^{-1+\Re \omega}\right)
$$

and

$$
R_{2}^{(2)}\left(z ; N_{0}, N_{1}, N_{2}\right)=\mathcal{O}\left(e^{-3|z|} z^{-1-\Re \omega}\right)
$$

\section{General levels}

The pattern of resubstituting into the successive remainder terms now is clear, and the general results can be written down by inspection and verified by induction. To facilitate the statement of the final theorems, we introduce the following notation pertaining to the parity of $l: \nu_{l}=0$ or 1 , according as $l$ is even or odd.

Theorem 7.1. Let $l$ be an arbitrary nonnegative integer and $N_{0}, N_{1}, \ldots, N_{l}$ be integers such that

$$
N_{j}=\beta_{j}|z|+\alpha_{j}, \quad j=0,1, \ldots, l,
$$

in which the $\beta$ 's are constants that satisfy

$$
0<\beta_{l}<\beta_{l-1}<\cdots<\beta_{0}
$$

and the $\alpha$ 's are bounded as $|z| \rightarrow \infty$. Then

$$
\begin{aligned}
u_{1}(z)= & \sum_{s=0}^{N_{0}-1} \frac{a_{s, 1}}{z^{s}}+(-)^{N_{0}} \frac{K_{1}}{z^{N_{0}-1}} \sum_{s=0}^{N_{1}-1} a_{s, 2} G_{\omega}^{(1)}\left(z ; N_{0}-s\right)+\ldots \\
& +(-)^{N_{0}+N_{1}+\cdots+N_{l-1}} \frac{K_{1}^{\frac{1}{2}\left(l+\nu_{l}\right)} K_{2}^{\frac{1}{2}\left(l-\nu_{l}\right)}}{z^{N_{0}-1}} \times \\
& \times \sum_{s=0}^{N_{l}-1} a_{s, 1+\nu_{l}} G_{\omega}^{(l)}\left(z ; N_{0}-N_{1}, \ldots, N_{l-2}-N_{l-1}, N_{l-1}-s\right) \\
& +R_{1}^{(l)}\left(z ; N_{0}, N_{1}, \ldots, N_{l}\right), \\
u_{2}(z)= & \sum_{s=0}^{N_{0}-1} \frac{a_{s, 2}}{z^{s}}+(-)^{N_{0}} \frac{K_{2}}{z^{N_{0}-1}} \sum_{s=0}^{N_{1}-1} a_{s, 1} G_{-\omega}^{(1)}\left(z ; N_{0}-s\right)+\ldots \\
+ & (-)^{N_{0}+N_{1}+\cdots+N_{l-1}} \frac{K_{1}^{\frac{1}{2}\left(l-\nu_{l}\right)} K_{2}^{\frac{1}{2}\left(l+\nu_{l}\right)}}{z^{N_{0}-1}} \times \\
& \times \sum_{s=0}^{N_{l}-1} a_{s, 2-\nu_{l}} G_{-\omega}^{(l)}\left(z ; N_{0}-N_{1}, \ldots, N_{l-2}-N_{l-1}, N_{l-1}-s\right) \\
+ & R_{2}^{(l)}\left(z ; N_{0}, N_{1}, \ldots, N_{l}\right)
\end{aligned}
$$


and as $z \rightarrow \infty$ in $|\mathrm{ph} z| \leqslant \pi$,

$$
\begin{aligned}
& R_{1}^{(l)}\left(z ; N_{0}, N_{1}, \ldots, N_{l}\right) \\
& \quad=\left[\prod_{j=0}^{l-1}\left\{\left(\beta_{j}-\beta_{j+1}\right)^{\beta_{j}-\beta_{j+1}} e^{\beta_{j+1}-\beta_{j}}\right\}^{|z|}\right]\left(\beta_{l}^{\beta_{l}} e^{-\beta_{l}}\right)^{|z|} \mathcal{O}\left(z^{\frac{1}{2}(1-l)+\nu_{l+1} \Re \omega}\right), \\
& R_{2}^{(l)}\left(z ; N_{0}, N_{1}, \ldots, N_{l}\right) \\
& \quad=\left[\prod_{j=0}^{l-1}\left\{\left(\beta_{j}-\beta_{j+1}\right)^{\beta_{j}-\beta_{j+1}} e^{\beta_{j+1}-\beta_{j}}\right\}^{|z|}\right]\left(\beta_{l}^{\beta_{l}} e^{-\beta_{l}}\right)^{|z|} \mathcal{O}\left(z^{\frac{1}{2}(1-l)-\nu_{l+1} \Re \omega}\right) .
\end{aligned}
$$

In Theorem 7.1, the remainder terms $R_{1}^{(l)}$ and $R_{2}^{(l)}$ are not optimized. Optimization is achieved on setting $\beta_{j}=l+1-j, j=0,1, \ldots, l$ or, equivalently, $N_{j}=(l+1-j) N$, where $N=|z|+\mathcal{O}(1)$. This yields:

Theorem 7.2. Let

$$
\begin{aligned}
u_{1}(z)= & \sum_{s=0}^{(l+1) N-1} \frac{a_{s, 1}}{z^{s}}+\sum_{j=1}^{l}(-)^{\frac{1}{2} j(j+1+2 l) N} \frac{K_{1}^{\frac{1}{2}\left(j+\nu_{j}\right)} K_{2}^{\frac{1}{2}\left(j-\nu_{j}\right)}}{z^{(l+1) N-1}} \times \\
& \times \sum_{s=0}^{(l+1-j) N-1} a_{s, 1+\nu_{j}} G_{\omega}^{(j)}(z ; N, \ldots, N,(l+2-j) N-s) \\
+ & R_{1}^{(l)}(z ;(l+1) N, l N, \ldots, N), \\
u_{2}(z)= & \sum_{s=0}^{(l+1) N-1} \frac{a_{s, 2}}{z^{s}}+\sum_{j=1}^{l}(-)^{\frac{1}{2} j(j+1+2 l) N} \frac{K_{1}^{\frac{1}{2}\left(j-\nu_{j}\right)} K_{2}^{\frac{1}{2}\left(j+\nu_{j}\right)}}{z^{(l+1) N-1}} \times \\
& \times \sum_{s=0}^{(l+1-j) N-1} a_{s, 2-\nu_{j}} G_{-\omega}^{(j)}(z ; N, \ldots, N,(l+2-j) N-s) \\
+ & R_{2}^{(l)}(z ;(l+1) N, l N, \ldots, N),
\end{aligned}
$$

where $N=|z|+\mathcal{O}(1)$. Then, as $z \rightarrow \infty$ in $|\mathrm{ph} z| \leqslant \pi$,

$$
\begin{aligned}
& R_{1}^{(l)}(z ;(l+1) N, l N, \ldots, N)=\mathcal{O}\left(e^{-(l+1)|z|} z^{-\frac{1}{2} l+\nu_{l+1} \Re \omega}\right), \\
& R_{2}^{(l)}(z ;(l+1) N, l N, \ldots, N)=\mathcal{O}\left(e^{-(l+1)|z|} z^{-\frac{1}{2} l-\nu_{l+1} \Re \omega}\right) .
\end{aligned}
$$

Remark (i). When $|\operatorname{ph} z| \leqslant \pi-\delta$, we can improve the estimates (7.6) by a factor $z^{-\frac{1}{2}}$ for all values of $l$ : we have only to retrace the analysis with the contour $\mathcal{P}$ freed from the indentations indicated in Figures 3.1 and 3.2 and apply the inequality (2.10).

Remark (ii). Each time the level $l$ increases by unity, the number of terms at each previous level in the expansions (7.3) increases by $|z|$ (approximately). In Remark (i) at the end of $\S 5$, we indicated how to optimize the remainder term at level 1 whilst constraining the number of terms at level 0 to be $|z|$. It transpired that $\frac{1}{2}|z|$ terms are needed at level 1 . This process may be continued. If we leave unchanged the number 
of terms at levels 0 and 1 , then $\frac{1}{4}|z|$ terms are needed at level 2 and so on, until we reach $2^{-l}|z|$ terms at level $l$. Furthermore, the remainder terms then satisfy

$$
\begin{aligned}
& R_{1}^{(l)}\left(z ; N_{0}, N_{1}, \ldots, N_{l}\right)=\mathcal{O}\left(2^{-L|z|} e^{-|z|} z^{\frac{1}{2}(1-l)+\nu_{l+1} \Re \omega}\right), \\
& R_{2}^{(l)}\left(z ; N_{0}, N_{1}, \ldots, N_{l}\right)=\mathcal{O}\left(2^{-L|z|} e^{-|z|} z^{\frac{1}{2}(1-l)-\nu_{l+1} \Re \omega}\right),
\end{aligned}
$$

where $L=2-2^{1-l}$. We observe immediately that $L<2$ for all values of $l$; in consequence the overall exponential improvement is limited to a factor $e^{-\gamma|z|}$, where $\gamma=1+2 \ln 2=2.386 \ldots$

The pattern of remainder terms just described was discovered by Berry and Howls [1] in a related problem. Some further observations on their paper will be found in the concluding section $\S 11$.

\section{Numerical stability}

Theorem 7.2 is an elegant result. Its essential message is that we can generate a relative error term whose asymptotic estimate for large $|z|$ contains a factor $e^{-(l+1)|z|}$, $l$ being an arbitrary nonnegative integer, by taking (approximately) $(l+1)|z|$ terms in the original Poincaré expansion, $l|z|$ terms in the first level re-expansion and so on, ending with $|z|$ terms at the $l$ th level. Furthermore, the coefficients of the auxiliary functions $G_{ \pm \omega}^{(l)}$ at successive levels alternate between the sets of coefficients in the original Poincaré expansions. In applications, however, there is a potential source of weakness. After we pass beyond the smallest term at any level, succeeding terms at that level grow rapidly. If any rises in absolute value well above unity (unity being the order of magnitude of $u_{1}(z)$ and $u_{2}(z)$ ), then severe cancellation will take place in summing the series (7.3) numerically, rendering the whole process unstable.

In order to demonstrate, and then overcome, this instability, we revert to Theorem 7.1 and permit the constants $\beta_{0}, \beta_{1}, \ldots, \beta_{l}$ to be arbitrary, subject to (7.2).

Consider first the sum at the zeroth level for $u_{1}(z)$. From (3.17a), we have

$$
a_{s, 1} \sim(-)^{s} K_{1} \Gamma(s+\omega), \quad s \rightarrow \infty .
$$

Accordingly (as we already know), as $s$ increases, $\left|a_{s, 1} z^{-s}\right|$ decreases until $s=|z|$, approximately, and it then increases unboundedly. For $s=N_{0}-1$ we have

$$
a_{N_{0}-1,1} \sim(-)^{N_{0}-1} K_{1} \Gamma\left(N_{0}-1+\omega\right)
$$

and, hence, by application of (7.1) and Stirling's formula,

$$
\left|a_{N_{0}-1,1} z^{1-N_{0}}\right| \sim \text { constant } \times\left(\beta_{0}^{\beta_{0}}\right)^{|z|} e^{-\beta_{0}|z|} z^{-\frac{1}{2}+\Re \omega}
$$

This estimate obviously exceeds, or is less than, unity, according as $\beta_{0}^{\beta_{0}} e^{-\beta_{0}} \gtrless 1$, that is, $\beta_{0} \gtrless e$.

Next, consider level 1. We have

$$
G_{\omega}^{(1)}\left(z ; M_{0}\right)=\Gamma\left(M_{0}+\Re \omega\right) \mathcal{O}(1)
$$

as $z \rightarrow \infty$ in $|\operatorname{ph} z| \leqslant \pi$, uniformly for all values of $M_{0}$ that satisfy $M_{0}+|\Re \omega| \geqslant 1$. This result may be proved by the method used to establish (4.6). With the aid of (3.17b), we see that

$$
a_{s, 2} G_{\omega}^{(1)}\left(z ; N_{0}-s\right)=\Gamma\{\max (s-\Re \omega, 1)\} \Gamma\left(N_{0}-s+\Re \omega\right) \mathcal{O}(1),
$$


uniformly for $s=0,1, \ldots, N_{1}-1$. From (7.1) and (8.1), we observe that at $s=0$ this estimate equals $a_{N_{0}-1,1} \mathcal{O}(z)$. As $s$ increases, the right-hand side of (8.4) decreases until $s=\frac{1}{2} N_{0}$, approximately, and then grows. Moreover, at $s=N_{1}-1$ we obtain

$$
a_{N_{1}-1,2} G_{\omega}^{(1)}\left(z ; N_{0}-N_{1}+1\right)=\Gamma\left(N_{1}-1-\Re \omega\right) \Gamma\left(N_{0}-N_{1}+1+\Re \omega\right) \mathcal{O}(1)
$$

and hence by use of (7.1) and Stirling's formula

$$
a_{N_{1}-1,2} G_{\omega}^{(1)}\left(z: N_{0}-\Lambda_{1}+1\right) z^{1-N_{0}}=b_{1}^{|z|} e^{-\beta_{0}|z|} \mathcal{O}(1),
$$

where

$$
b_{1}=\left(\beta_{0}-\beta_{1}\right)^{\beta_{0}-\beta_{1}} \beta_{1}^{\beta_{1}} .
$$

Since $\beta_{0}-\beta_{1}$ and $\beta_{1}$ are both less then $\beta_{0}$, we have $b_{1}<\beta_{0}^{\beta_{0}}$. Accordingly, the estimate (8.5) is asymptotically small compared with (8.2).

At higher levels

$$
\begin{aligned}
& G_{\omega}^{(l)}(z ;\left.N_{0}-N_{1}, \ldots, N_{l-2}-N_{l-1}, N_{l-1}-s\right) \\
&= \Gamma\left(N_{0}-N_{1}+\Re \omega\right) \cdots \Gamma\left(N_{l-2}-N_{l-1}+(-)^{l-2} \Re \omega\right) \times \\
& \quad \times \Gamma\left(N_{l-1}-s+(-)^{l-1} \Re \omega\right) \mathcal{O}(1),
\end{aligned}
$$

as $z \rightarrow \infty$ in $\mid$ ph $z \mid \leqslant \pi$, uniformly for $s \in\left[0, N_{l}-1\right]$. As in the case of (8.3), this result may be proved by the method used to establish (4.6), (5.13), and (6.10). Armed with this estimate, we may show that the behavior of the terms at each level follows a similar pattern. The first term is within a factor $\mathcal{O}(z)$ of the last term at the previous level. As $s$ increases the terms at level $l$ decrease to a minimum at $s=\frac{1}{2} N_{l-1}$, approximately, and then grow in magnitude. Moreover, whether or not the series at this level is terminated at this minimum, as long as $0<\beta_{l}<\beta_{l-1}$, the last term is asymptotically small compared with $\left|a_{N_{0}-1,1} z^{1-N_{0}}\right|$.

A similar analysis applies to the expansion (7.3b); basically we have only to replace $\Re \omega$ by $-\Re \omega$ throughout. We therefore conclude: whatever the value of the constants $\beta_{1}, \beta_{2}, \ldots, \beta_{l}$, as long as (7.2) applies, the summation of the series (7.3) is numerically stable or unstable according as $\beta_{0} \lessgtr e$.

In the remaining case, given by $\beta_{0}=e$, the growth (if any) of $a_{N_{0}-1,1} z^{1-N_{0}}$ and $a_{N_{0}-1,2} z^{1-N_{0}}$ is algebraic rather than exponential; compare (8.2). Accordingly, we may say that the summation of (7.3) is weakly unstable, in the sense that any numerical cancellation that takes place will not be catastrophic in nature.

On returning to Theorem 7.2, we note that $\beta_{0}=l+1$. Hence, Theorem 7.2 leads to a stable summation procedure only when $l=0$ or 1 , that is, when the series (7.3) are terminated at the zeroth or first level.

When $l \geqslant 2$ we need to modify the optimization of the remainder term in order to maintain stability. This means that with $\beta_{0}=e$ we need to minimize

$$
\left[\prod_{j=0}^{l-1}\left(\beta_{j}-\beta_{j+1}\right)^{\beta_{j}-\beta_{j+1}} e^{\beta_{j+1}-\beta_{j}}\right]\left(\beta_{l}^{\beta_{l}} e^{-\beta_{l}}\right)
$$

compare (7.4). It is easily verified that this minimum occurs when

$$
\beta_{j}=\frac{l+1-j}{l+1} e, \quad j=1,2, \ldots, l
$$

and it equals $(l+1)^{-e}$. 
We may summarize the findings of this section as follows:

Theorem 8.1 (Numerically stable expansion). Assume the notation of Theorem 7.1 and that $l \geqslant 2$. If

$$
N_{j}=\frac{l+1-j}{l+1} e|z|+\mathcal{O}(1), \quad j=0,1, \ldots, l
$$

then as $z \rightarrow \infty$ in $|\mathrm{ph} z| \leqslant \pi$

$$
\begin{aligned}
& R_{1}^{(l)}\left(z ; N_{0}, N_{1}, \ldots, N_{l}\right)=\mathcal{O}\left\{(l+1)^{-e|z|} z^{\frac{1}{2}(1-l)+\nu_{l+1} \Re \omega}\right\} \\
& R_{2}^{(l)}\left(z ; N_{0}, N_{1}, \ldots, N_{l}\right)=\mathcal{O}\left\{(l+1)^{-e|z|} z^{\frac{1}{2}(1-l)-\nu_{l+1} \Re \omega}\right\} .
\end{aligned}
$$

Remark. When $l \geqslant 2$ the overall exponential improvement yielded by Theorem 8.1 is less than that yielded by Theorem 7.2 , especially when $l \geqslant 3$. On the other hand, fewer terms are needed in the expansions (7.3).

\section{Example}

We take as example the differential equation

$$
\frac{d^{2} w}{d z^{2}}+\left(\frac{1}{z}-1\right) \frac{d w}{d z}-\frac{1}{2 z} w=0
$$

satisfied by $e^{\frac{1}{2} z} K_{0}\left(\frac{1}{2} z\right)$ and $e^{\frac{1}{2} z} K_{0}\left(\frac{1}{2} z e^{-\pi i}\right)$, where $K_{0}$ is the modified Bessel function. The values of $\lambda_{1}, \lambda_{2}, \mu_{1}, \mu_{2}$, and $\omega$ are found to be $\lambda_{1}=0, \lambda_{2}=1, \mu_{1}=\mu_{2}=-\frac{1}{2}$, and $\omega=0$. The basic functions $u_{1}(z), u_{2}(z)$ and the coefficients $a_{s, 1}, a_{s, 2}$ are given by $^{4}$

and

$$
u_{1}(z)=u_{2}(z)=\pi^{-\frac{1}{2}} z^{\frac{1}{2}} e^{\frac{1}{2} z} K_{0}(z / 2)
$$

$$
a_{s, 1}=a_{s, 2}=(-)^{s} \frac{1^{2} \cdot 3^{2} \cdots(2 s-1)^{2}}{4^{s} s !}
$$

and the coefficients $K_{1}$ and $K_{2}$ in the connection formulas (3.10) are known to be

$$
K_{1}=K_{2}=\pi^{-1}
$$

see, for example, [8], Chapter $7, \S \S 4,8$, or we can take the limit as $s \rightarrow \infty$ in (3.17) above, using (9.2).

For illustration, we take $z=20$. The squares in Figure 9.1 are the plot of the logarithm to base 10 of the absolute value of each term in the expansions (7.3) against its ordinal number. ${ }^{5}$

For $l=0$ we set $N_{0}=20$, which terminates the original asymptotic expansions (3.8) at (or very nearly at) their smallest term. For $l=1$ we set $N_{0}=40, N_{1}=20$, and for $l=2$ we set $N_{0}=60, N_{1}=40, N_{2}=20$. As expected, in the case $l=2$ a few of the terms rise above unity in magnitude, indicating some instability in the summation. For $l=3$ (not shown in the diagram) the instability would be much more severe.

\footnotetext{
${ }^{4}$ Another solution of (9.1) is $e^{\frac{1}{2} z} I_{0}\left(\frac{1}{2} z\right)$, where $I_{0}$ is the other modified Bessel function. Although $\pi^{\frac{1}{2}} e^{\frac{1}{2} z} I_{0}\left(\frac{1}{2} z\right)$ has the same form of asymptotic expansion as $-i \pi^{-\frac{1}{2}} e^{\frac{1}{2} z} K_{0}\left(\frac{1}{2} z e^{-\pi i}\right)$, it should be noted that it cannot be taken as $w_{2}(z)$ because $\pi^{\frac{1}{2}} e^{\frac{1}{2} z} I_{0}\left(\frac{1}{2} z\right)$ is not a recessive solution of (9.1) in the sector $\frac{1}{2} \pi+\delta \leqslant \operatorname{ph} z \leqslant \frac{3}{2} \pi-\delta$ (or for that matter in any sector).

${ }^{5}$ The terms were computed by the methods given in the Appendix of [1].
} 


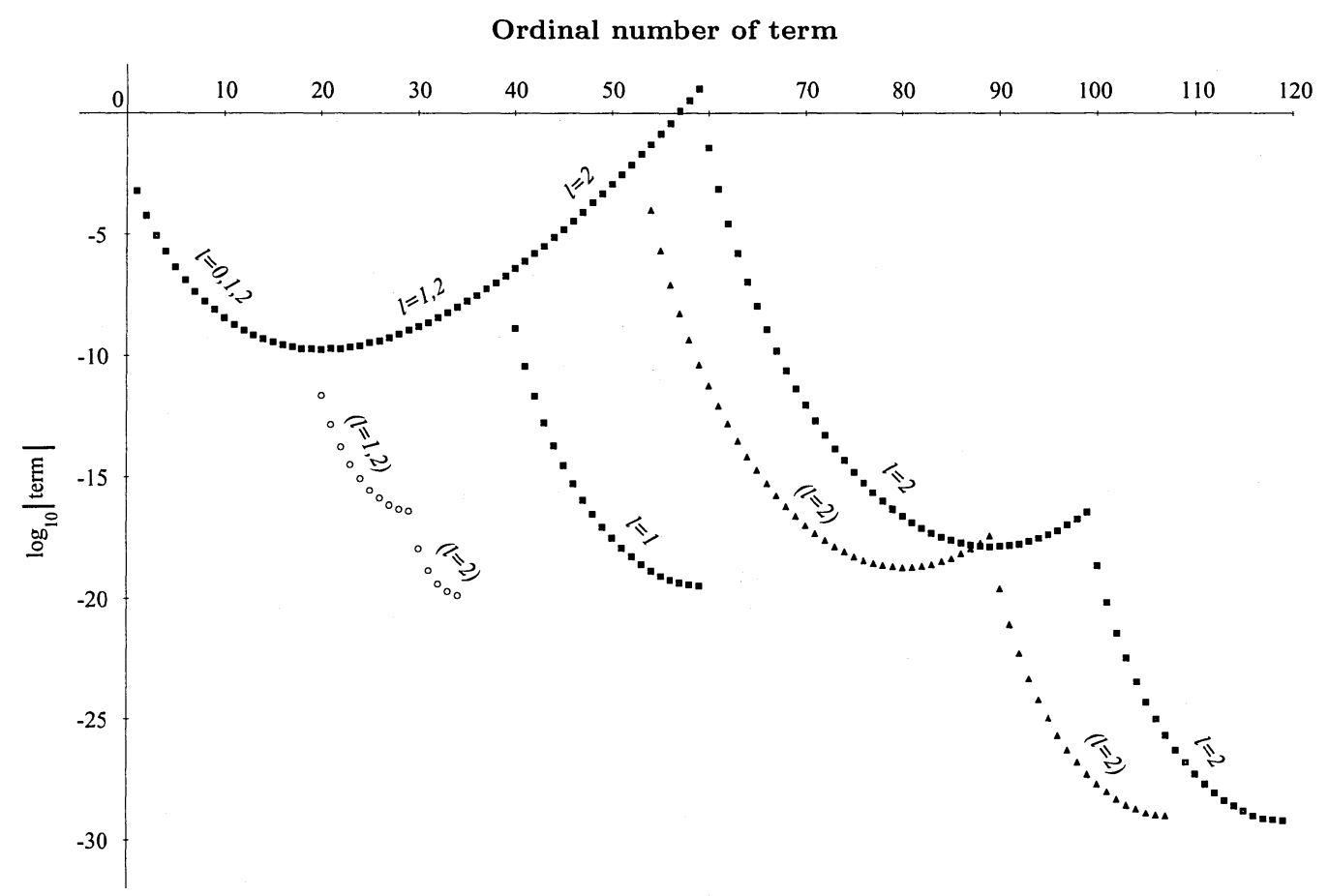

Figure 9.1. Magnitudes of the terms in the three hyperasymptotic expansions.

The curve indicated by the triangles shows the modification of the case $l=2$ consequent upon using the numerically stable form of expansion furnished by Theorem 8.1. We take $N_{0}=54, N_{1}=36, N_{2}=18$. In this expansion all terms are below unity in magnitude, but as expected the final accuracy is slightly less; see Table 9.1.

Next, the curve indicated by the circles is for the case $l=2$, with $N_{0}=20, N_{1}=10$, $N_{2}=5$. This corresponds to optimization with a fixed number of terms at each level.

\begin{tabular}{|c|c|c|c|c|c|}
\hline level & approximation & exact-approx. & $N_{0}$ & $N_{1}$ & $N_{2}$ \\
\hline 0 & 0.988139270254112817375114072700 & $1.8 \times 10^{-10}$ & 20 & & \\
1 & 0.988139270438649159234772899885 & $3.4 \times 10^{-20}$ & 40 & 20 & \\
2 & 0.988139270438649159268429546441 & $6.1 \times 10^{-30}$ & 60 & 40 & 20 \\
\hline 0 & 0.988139270254112817375114072700 & $1.8 \times 10^{-10}$ & 20 & & \\
1 & 0.988139270438649159234772899885 & $3.4 \times 10^{-20}$ & 40 & 20 & \\
2 & 0.988139270438649159268429546437 & $1.0 \times 10^{-29}$ & 54 & 36 & 18 \\
\hline 0 & 0.988139270254112817375114072700 & $1.8 \times 10^{-10}$ & 20 & & \\
1 & 0.988139270438649118161993331991 & $4.1 \times 10^{-18}$ & 20 & 10 & \\
2 & 0.988139270438649159281542952765 & $-1.3 \times 10^{-20}$ & 20 & 10 & 5 \\
\hline exact & 0.988139270438649159268429546447 & 0 & & & \\
\hline
\end{tabular}

TABLE 9.1. Hyperasymptotic approximations to $\pi^{-\frac{1}{2}} z^{\frac{1}{2}} e^{\frac{1}{2} z} K_{0}\left(\frac{1}{2} z\right)$ for $z=20$. 
As we noted in Remark (ii) at the end of $\S 7$, when continued to higher levels the last term can never be less than about $e^{-20(1+2 \ln 2)} \fallingdotseq 2 \times 10^{-21}$. This is approximately $10^{8}$ times as large as the last term in the other two expansions with $l=2$.

Lastly, we observe that the pattern of growth depicted by all the curves in Figure 9.1 is exactly that predicted in $\S 8$ for the general case.

\section{Extensions of the regions of validity}

The region of validity supplied by Theorems 7.1, 7.2, and 8.1 is the closed sector $|\operatorname{ph} z| \leqslant \pi$. Values of the analytic continuation of $w_{1}(z)$ and $w_{2}\left(z e^{\pi i}\right)$ for any other value of $\mathrm{ph} z$ can be calculated by repeated application of the connection formulas (3.6) or (3.9). Nevertheless, it is of some interest to ascertain whether the region of validity in Theorems 7.2 and 8.1 can be extended beyond ph $z= \pm \pi$, especially as an extension to $|\operatorname{ph} z| \leqslant \frac{5}{2} \pi-\delta$ was supplied in [6] for the unoptimized case at level 1 . We shall show in this section that such extensions can be made at all levels, although at the cost of weakening the asymptotic estimates of the remainder terms. We shall achieve this by constructing continuation formulas for the remainder terms.

From (3.9) and (3.12) we derive

$$
\begin{aligned}
R_{1}^{(0)}\left(z ; N_{0}\right)-R_{1}^{(0)}\left(z e^{-2 \pi i} ; N_{0}\right) & =u_{1}(z)-u_{1}\left(z e^{-2 \pi i}\right) \\
& =2 \pi i K_{1} e^{-\omega \pi i} e^{z} z^{\omega} u_{2}\left(z e^{-\pi i}\right) .
\end{aligned}
$$

If $\pi \leqslant \operatorname{ph} z \leqslant \frac{3}{2} \pi$ and $N_{0}=|z|+\mathcal{O}(1)$, then from (4.11a) we have

$$
R_{1}^{(0)}\left(z e^{-2 \pi i} ; N_{0}\right)=\mathcal{O}\left(e^{-|z|} z^{\Re \omega}\right)
$$

whereas from $(3.8 \mathrm{~b})$

$$
2 \pi i K_{1} e^{-\omega \pi i} e^{z} z^{\omega} u_{2}\left(z e^{-\pi i}\right)=\mathcal{O}\left(e^{z} z^{\Re \omega}\right)
$$

Hence,

$$
R_{1}^{(0)}\left(z ; N_{0}\right)=\mathcal{O}\left(e^{z} z^{\Re \omega}\right), \quad \pi \leqslant \operatorname{ph} z \leqslant \frac{3}{2} \pi-\delta
$$

Similarly,

$$
R_{2}^{(0)}\left(z ; N_{0}\right)=\mathcal{O}\left(e^{z} z^{-\Re \omega}\right), \quad \pi \leqslant \operatorname{ph} z \leqslant \frac{3}{2} \pi-\delta
$$

Accordingly, the exponential improvement supplied by (4.11) deteriorates gradually as we pass beyond ph $z=\pi$ and disappears altogether as we approach $\mathrm{ph} z=\frac{3}{2} \pi$.

At the next level, we have from (5.3)

$$
\begin{aligned}
& R_{1}^{(1)}\left(z ; N_{0}, N_{1}\right)-R_{1}^{(1)}\left(z e^{-2 \pi i} ; N_{0}, N_{1}\right)=R_{1}^{(0)}\left(z ; N_{0}\right)-R_{1}^{(0)}\left(z e^{-2 \pi i} ; N_{0}\right) \\
& \quad+(-)^{N_{0}-1} \frac{K_{1}}{z^{N_{0}-1}} \sum_{s=0}^{N_{1}-1} a_{s, 2}\left\{G_{\omega}^{(1)}\left(z ; N_{0}-s\right)-G_{\omega}^{(1)}\left(z e^{-2 \pi i} ; N_{0}-s\right)\right\}
\end{aligned}
$$


From (10.1) and (3.12b), with $z$ and $N_{0}$ replaced by $z e^{-\pi i}$ and $N_{1}$, respectively, we obtain

$R_{1}^{(0)}\left(z ; N_{0}\right)-R_{1}^{(0)}\left(z e^{-2 \pi i} ; N_{0}\right)=2 \pi i K_{1} e^{-\omega \pi i} e^{z} z^{\omega}\left\{\sum_{s=0}^{N_{1}-1}(-)^{s} \frac{a_{s, 2}}{z^{s}}+R_{2}^{(0)}\left(z e^{-\pi i} ; N_{1}\right)\right\}$

Also, from (2.9) with $l=1$ and $M_{0}=N_{0}-s$, and using (2.2a), we have

$$
\begin{gathered}
(-)^{N_{0}} \frac{K_{1}}{z^{N_{0}-1}} \sum_{s=0}^{N_{1}-1} a_{s, 2}\left\{G_{\omega}^{(1)}\left(z ; N_{0}-s\right)-G_{\omega}^{(1)}\left(z e^{-2 \pi i} ; N_{0}-s\right)\right\} \\
\quad=2 \pi i K_{1} e^{-\omega \pi i} e^{z} z^{\omega} \sum_{s=0}^{N_{1}-1}(-)^{s} \frac{a_{s, 2}}{z^{s}} .
\end{gathered}
$$

On substituting into (10.5) by means of the last two equations, we find that

$$
R_{1}^{(1)}\left(z ; N_{0}, N_{1}\right)-R_{1}^{(1)}\left(z e^{-2 \pi i} ; N_{0}, N_{1}\right)=2 \pi i K_{1} e^{-\omega \pi i} e^{z} z^{\omega} R_{2}^{(0)}\left(z e^{-\pi i} ; N_{1}\right) .
$$

Once again, by symmetry

$$
R_{2}^{(1)}\left(z ; N_{0}, N_{1}\right)-R_{2}^{(1)}\left(z e^{-2 \pi i} ; N_{0}, N_{1}\right)=2 \pi i K_{2} e^{\omega \pi i} e^{z} z^{-\omega} R_{1}^{(0)}\left(z e^{-\pi i} ; N_{1}\right)
$$

Suppose that $\pi \leqslant \operatorname{ph} z \leqslant 2 \pi, N_{0}=2|z|+\mathcal{O}(1)$ and $N_{1}=|z|+\mathcal{O}(1)$. Then from (5.19) we have

$$
R_{1}^{(1)}\left(z e^{-2 \pi i} ; N_{0}, N_{1}\right)=\mathcal{O}\left(e^{-2|z|} z^{-\frac{1}{2}}\right)
$$

and from (4.11b)

$$
2 \pi i K_{1} e^{-\omega \pi i} e^{z} z^{\omega} R_{2}^{(0)}\left(z e^{-\pi i} ; N_{1}\right)=\mathcal{O}\left(e^{z-|z|}\right)
$$

Combination of (10.6a), (10.7), and (10.8) yields

$$
R_{1}^{(1)}\left(z ; N_{0}, N_{1}\right)=\mathcal{O}\left(e^{z-|z|}\right), \quad \pi \leqslant \operatorname{ph} z \leqslant 2 \pi .
$$

Next, if $2 \pi \leqslant \operatorname{ph} z \leqslant \frac{5}{2} \pi-\delta$ and $N_{0}=2|z|+\mathcal{O}(1), N_{1}=|z|+\mathcal{O}(1)$, then (10.7) continues to apply, but from $(10.4 \mathrm{~b})$ we see that the right-hand side of $(10.8)$ is replaced by $\mathcal{O}(1)$. Accordingly,

$$
R_{1}^{(1)}\left(z ; N_{0}, N_{1}\right)=\mathcal{O}(1), \quad 2 \pi \leqslant \operatorname{ph} z \leqslant \frac{5}{2} \pi-\delta .
$$

By similar analysis we may show that the estimates (10.7), (10.9), and (10.10) also apply to the remainder term $R_{2}^{(1)}\left(z ; N_{0}, N_{1}\right)$ in the same ranges of $\mathrm{ph} z$.

The process may be continued to higher levels and also to negative values of $\mathrm{ph} z$. With the aid of (2.9), (3.9), and (7.3), the generalizations of (10.6) may be verified to be

$$
\begin{array}{r}
R_{1}^{(l)}\left(z ; N_{0}, N_{1}, \ldots, N_{l}\right)-R_{1}^{(l)}\left(z e^{-2 \pi i} ; N_{0}, N_{1}, \ldots, N_{l}\right) \\
\quad=2 \pi i K_{1} e^{-\omega \pi i} e^{z} z^{\omega} R_{2}^{(l-1)}\left(z e^{-\pi i} ; N_{1}, N_{2}, \ldots, N_{l}\right)
\end{array}
$$


and

$$
\begin{aligned}
& R_{2}^{(l)}\left(z ; N_{0}, N_{1}, \ldots, N_{l}\right)-R_{2}^{(l)}\left(z e^{-2 \pi i} ; N_{0}, N_{1}, \ldots, N_{l}\right) \\
& \quad=2 \pi i K_{2} e^{\omega \pi i} e^{z} z^{-\omega} R_{1}^{(l-1)}\left(z e^{-\pi i} ; N_{1}, N_{2}, \ldots, N_{l}\right) .
\end{aligned}
$$

Then by induction we may establish the following result.

Theorem 10.1. If $k=0,1, \ldots, l$, then as $z \rightarrow \infty$ in $k \pi \leqslant|\operatorname{ph} z| \leqslant(k+1) \pi$, the remainder terms in Theorem 7.2 satisfy

$$
\begin{aligned}
& R_{1}^{(l)}(z ;(l+1) N, l N, \ldots, N)=\mathcal{O}\left(e^{\nu_{k} z+(k-l-1)|z|} z^{\frac{1}{2}(k-l)+\nu_{l+1} \Re \omega}\right), \\
& R_{2}^{(l)}(z ;(l+1) N, l N, \ldots, N)=\mathcal{O}\left(e^{\nu_{k} z+(k-l-1)|z|} z^{\frac{1}{2}(k-l)-\nu_{l+1} \Re \omega}\right) .
\end{aligned}
$$

Also, if $z \rightarrow \infty$ in $(l+1) \pi \leqslant|\operatorname{ph} z| \leqslant\left(l+\frac{3}{2}\right) \pi-\delta$, then

$$
\begin{aligned}
& R_{1}^{(l)}(z ;(l+1) N, l N, \ldots, N)=\mathcal{O}\left(e^{\nu_{l+1} z} z^{\nu_{l+1} \Re \omega}\right), \\
& R_{2}^{(l)}(z ;(l+1) N, l N, \ldots, N)=\mathcal{O}\left(e^{\nu_{l+1} z} z^{-\nu_{l+1} \Re \omega}\right) .
\end{aligned}
$$

Remark. The sector of validity of Theorem 8.1 can also be extended in a similar manner. However, because the estimates (8.8) involve the factor $\exp \{-e|z| \ln (l+1)\}$ instead of the more powerful factor $\exp \{-(l+1)|z|\}$, the increase is more restrictive. We shall not enter into details.

\section{Conclusions}

There are several points of similarity between this paper and the paper of Berry and Howls [1]. Thus the same hyperterminants appear in the expansions, and the coefficients of these hyperterminants are related to the coefficients at the lowest level. There are significant differences, however. In the first place, the theory in [1] is purely formal: it is based on Dingle's re-expansions of remainder terms using Borel summation and a "resurgence formula", also due to Dingle [3]. Secondly, the asymptotic expansions that are re-expanded in [1] are the Liouville-Green expansions. Only in certain cases, for example, Airy's differential equation, are these the same as the Poincaré power-series expansions. Thirdly, the optimization process is carried out differently, in that the number of terms at a given level is constrained not to change when higher levels are incorporated into the expansion. As we saw in Remark (ii) of $\S 7$, this results in there being (approximately) $2^{-j}|z|$ terms at level $j$, and whatever the final level $l$ happens to be the overall exponential improvement in the estimate for the error term is restricted to $e^{-(1+2 \ln 2)|z|}$, that is, $e^{-(2.386 \ldots)|z|}$ times a power of $|z|$. With our method of optimization, there is no limit on the attainable exponential improvement, even in the numerically stable form.

In earlier papers, for example [6], expansions at level 1 were regarded as providing a smooth interpretation of the Stokes phenomenon, because of the behavior and analytic nature of the individual terms in the neighborhoods of the Stokes lines. Since each branch of each $G_{\omega}^{(l)}$ function is analytic in its argument $z$ (except at the origin), the hyperasymptotic expansions also furnish a smooth interpretation of the Stokes phenomenon. An added feature, however, is that these expansions apply to several 
Stokes lines simultaneously. Thus, for example, $2 l$ Stokes lines are covered in this way in the region of validity given by Theorem 10.1 .

The practical implementation of the new expansions, in common with those of [1], depends on the ability to compute the necessary hyperterminants either by means of asymptotic approximations or by direct numerical methods. At the $l$ th level, the hyperterminants are $l$-fold multiple integrals. The case $l=0$ is trivial, and for $l=1$, algorithms and software packages exist; see $[4, \S 5.7] .{ }^{6}$ Some progress on the problem of computing hyperterminants at level 2 in the case when $\omega$ is an integer is made in Appendix B of [1], and these results were used in the numerical example given in $\S 9$.

Acknowledgement. The authors are indebted to the referees for helpful suggestions in the presentation of this paper.

\section{References}

1. M. V. Berry and C. J. Howls, Hyperasymptotics, Proc. Roy. Soc. London, Ser. A 430 (1990), 653-668.

2. - Hyperasymptotics for integrals with saddles, Proc. Roy. Soc. London, Ser. A 434 (1991), 657-675.

3. R. B. Dingle, Asymptotic Expansions: Their Derivation and Interpretation, Academic Press, London, 1973.

4. D. W. Lozier and F. W. J. Olver, Numerical evaluation of special functions. In Mathematics of Computation 1943-1993: A Half-Century of Computational Mathematics, (W. Gautschi, ed.), Proceedings of Symposia in Applied Mathematics, Vol. 48, American Mathematical Society, Providence, Rhode Island, 1994.

5. A. B. Olde Daalhuis, Hyperasymptotic expansions of confluent hypergeometric functions, IMA J. Appl. Math. 49 (1992), 203-216.

6. A. B. Olde Daalhuis and F. W. J. Olver, Exponentially-improved asymptotic solutions of ordinary differential equations. II: Irregular singularities of rank one, Proc. Roy. Soc. London, Ser. A 445 (1994), 39-56.

7. , On the calculation of Stokes multipliers for linear differential equations of the second order, Methods Appl. Anal. (to appear).

8. F. W. J. Olver, Asymptotics and Special Functions, Academic Press, New York, 1974.

9. __ Exponentially-improved asymptotic solutions of ordinary differential equations I: The confluent hypergeometric function, SIAM J. Math. Anal. 24 (1993), 756-767.

Institute for Physical Science and Technology, University of Maryland, College Park, MARYLAND 20742, U.S.A.

E-mail: aod@olgao.umd.edu, olver@bessel.umd.edu

\footnotetext{
${ }^{6}$ At least one existing software package is defective in the complex plane, however, especially near the Stokes lines ph $z= \pm \pi$.
} 